This item was submitted to Loughborough's Research Repository by the author.

Items in Figshare are protected by copyright, with all rights reserved, unless otherwise indicated.

\title{
Source term estimation of a hazardous airborne release using an unmanned aerial vehicle
}

PLEASE CITE THE PUBLISHED VERSION

https://doi.org/10.1002/rob.21844

\section{PUBLISHER}

(c) Wiley

\section{VERSION}

AM (Accepted Manuscript)

\section{PUBLISHER STATEMENT}

This is the peer reviewed version of the following article: HUTCHINSON, M., LIU, C. and CHEN, W-H., 2019. Source term estimation of a hazardous airborne release using an unmanned aerial vehicle. Journal of Field Robotics, 36(4), pp. 797-817, which has been published in final form at https://doi.org/10.1002/rob.21844. This article may be used for non-commercial purposes in accordance with Wiley Terms and Conditions for Use of Self-Archived Versions.

\section{LICENCE}

CC BY-NC-ND 4.0

\section{REPOSITORY RECORD}

Hutchinson, Michael, Cunjia Liu, and Wen-Hua Chen. 2018. "Source Term Estimation of a Hazardous Airborne Release Using an Unmanned Aerial Vehicle”. figshare. https://hdl.handle.net/2134/35756. 


\title{
Source term estimation of a hazardous airborne release using an unmanned aerial vehicle
}

\author{
Michael Hutchinson* \\ Department of Aeronautical and Automotive Engineering \\ Loughborough University \\ Leicestershire, United Kingdom \\ M. Hutchinson2@1boro.ac.uk \\ Cunjia Liu \\ Department of Aeronautical and Automotive Engineering \\ Loughborough University \\ Leicestershire, United Kingdom \\ C.Liu5@lboro.ac.uk \\ Wen-Hua Chen \\ Department of Aeronautical and Automotive Engineering \\ Loughborough University \\ Leicestershire, United Kingdom \\ W. Chen@lboro.ac.uk
}

\begin{abstract}
Gaining information about an unknown gas source is a task of great importance with applications in several areas including: responding to gas leaks or suspicious smells, quantifying sources of emissions, or in an emergency response to an industrial accident or act of terrorism. In this paper, a method to estimate the source term of a gaseous release using measurements of concentration obtained from an unmanned aerial vehicle (UAV) is described. The source term parameters estimated include the three dimensional location of

${ }^{*}$ Use footnote for providing further information about author (webpage, alternative address). Acknowledgments to funding agencies should go in the Acknowledgments section at the end of the paper.
\end{abstract}


the release, its emission rate, and other important variables needed to forecast the spread of the gas using an atmospheric transport and dispersion model. The parameters of the source are estimated by fusing concentration observations from a gas detector on-board the aircraft, with meteorological data and an appropriate model of dispersion. Two models are compared in this paper, both derived from analytical solutions to the advection diffusion equation. Bayes' theorem, implemented using a sequential Monte Carlo algorithm, is used to estimate the source parameters in order to take into account the large uncertainties in the observations and formulated models. The system is verified with novel, outdoor, fully automated experiments, where observations from the UAV are used to estimate the parameters of a diffusive source. The estimation performance of the algorithm is assessed subject to various flight path configurations and wind speeds. Observations and lessons learned during these unique experiments are discussed and areas for future research are identified.

\section{Introduction}

\subsection{Motivation}

Finding the source of a gas, knowing an estimate of its emission rate, forecasting the spatial extent of a gas, or even simply confirming the presence or absence of hazardous airborne material in an area has immense benefit in emergency response and several other applications. The gaseous release could be man-made or naturally occurring, hazardous to the environment or to human health, or provide clues as to the location of resources. Examples of well known naturally occurring releases include some sources of methane emissions or volcanic eruptions [Mastin et al., 2009]. It is of great interest to identify and quantify these sources in order to assess the environmental, social and commercial impact. Man-made releases are predominantly a result of industrial emissions [He et al., 2011] and accidents such as chemical spills [Thanabalasingham et al., 1991, Derezinski et al., 2003]. The accumulating effect on the environment and the increasing frequency of accidents makes the ability to monitor these emissions, or respond effectively to these accidents, of utmost importance. On the contrary, there is the rare event where a hazardous release could be deliberate, in the case of an act of terrorism [Okumura et al., 1998] or war. In these circumstances it would be important to perform a rapid, planned response to minimise casualties to the public and first response personnel.

Regardless of the specific application, estimating the source term of a release can provide important information and improved situational awareness. The source term incorporates important details consisting of the 
location of the release and the remaining parameters needed to model the spread of the material and forecast the future harm using atmospheric transport and dispersion (ATD) models [Hutchinson et al., 2017b]. These parameters include the release rate and potentially the start and stop times of the release which can provide valuable knowledge about the environmental impact and post hazard assessment or forensics.

Source term estimation is most commonly performed using an array of static sensors on the ground. Formulated as an inverse problem, Bayesian inference or optimisation algorithms are used to fuse point-wise concentration observations of the hazard with hypothesised predictions from a model [Hutchinson et al., 2017b]. Although there are some benefits, such as early detection in areas of interest with an existing network in place, static sensors have several drawbacks which are overcome by mobile deployment. These include the cost of maintenance, the quantity required, and their manual positioning which can be slow and provide less information than, for example, an intelligently guided unmanned aerial vehicle (UAV).

A UAV has the ability to sample from the most desirable locations to gather high quality spatial temporal data. This can enable rapid estimation of the location of the hazardous release and the other parameters of the source term. UAVs have the potential to monitor an area of interest, survey an area to confirm the presence or absence of hazardous material and locate the hazardous sources; all whilst keeping emergency responders out of the danger yet still provided with high quality information from the on-board sensors. This present work focuses on the development and testing of an unmanned aerial system to perform source term estimation in response to a release of hazardous material. In the future, it will be extended to conduct a more efficient information based search, and the resulting system is envisioned to feature several other capabilities to maximise situational awareness in an emergency scenario; such as a live video feed.

\subsection{System overview}

A reader unfamiliar with gas source localisation, atmospheric dispersion, or source term estimation research could, at first glance, perceive the problem as rather trivial. Instinctively, to localise a gas source, one would propose to trace the concentration gradient of the gas towards its origin. When in fact, it is an immensely challenging problem due to the random nature of turbulence and gas dispersion which can cause erratic fluctuations in concentration, resulting in sporadic, highly volatile readings from the gas detectors [Shraiman and Siggia, 2000, Vergassola et al., 2007]. Indeed, from a biological point of view, living organisms that adopt a gradient based, or "chemotaxis", approach are of a microscopic scale such as Escherichia Coli bacteria [Adler, 1966]. On a larger scale the procedure is abandoned. For the readers comprehension, an example plume on the scale of the experiments is shown in Fig 1. The coloured smoke was used to visualise the effect of the 


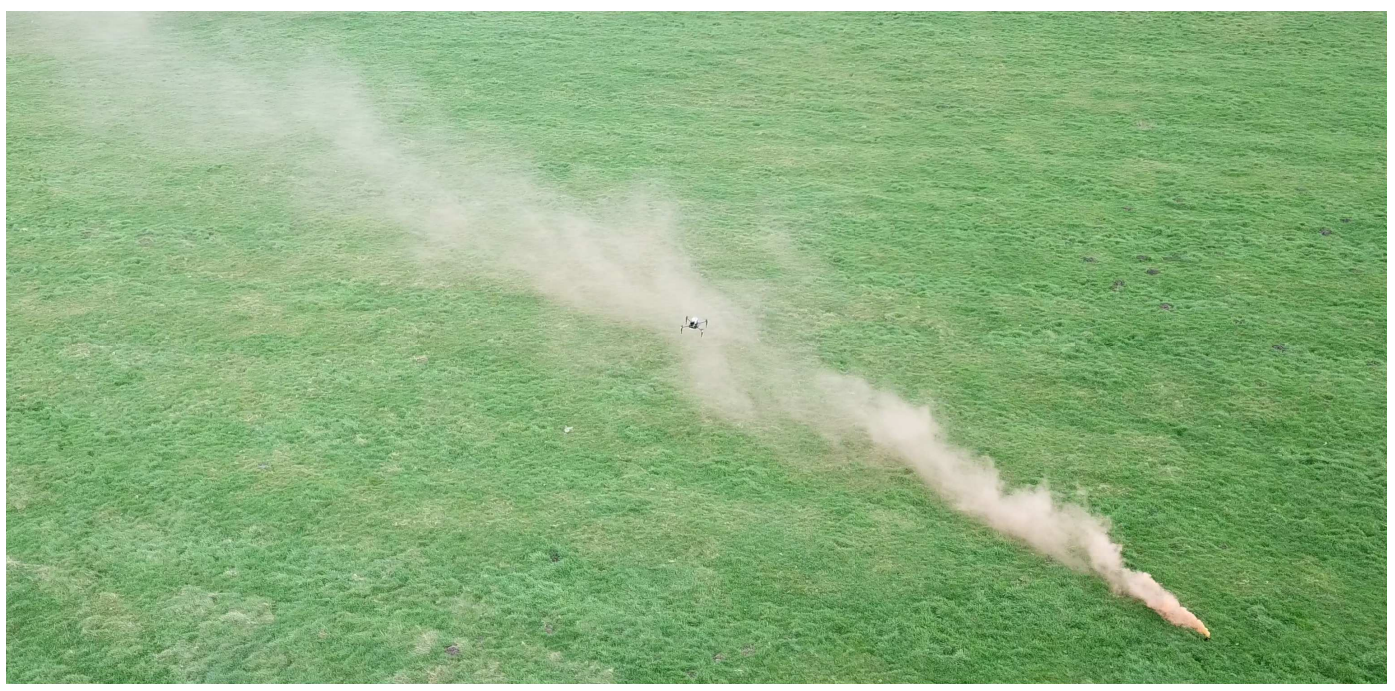

Figure 1: Visualisation of a UAV flying in the plume of a point source of emissions.

UAV (seen in the centre of the figure) on the gas dispersion. In this example, despite a strongly emitting source, gaps in the plume can be observed nonetheless.

To overcome this challenge, Bayes' theorem is proposed to estimate the parameters of the source given the noisy readings from the sensors, uncertainties in the meteorological conditions, and errors in the underlying models and assumptions therein. The system executes a systematic sweep search pattern and estimates the parameters of the hazardous release by fusing the information from a gas detector on-board the vehicle with meteorological information and an appropriate model of gas dispersion. Two models are compared using the data collected during the experimental trials conducted in this paper. The output of the system is an estimate of the source term parameters of the hazardous release which includes all the information required to forecast its dispersion from the source. Besides the ability to forecast the hazard, the estimated parameters provide other valuable information such as the location of the source and its rate of emission.

The system primarily consists of a quadrotor UAV and a laptop as a base station. The quadrotor is equipped with a Photoionisation Detector (PID) which is used to measure the concentrations of the hazardous gas. Currently, the measured concentrations are sent to the ground station using a 5Ghz WiFi network. The ground station will run the Bayesian estimation of the source parameters and send the next position demand to the UAV. This set-up is chosen to facilitate the development of a more efficient on-line planning algorithm in the future. Due to the very fast computational time of the algorithm the computation could potentially be performed by the on-board computer of the UAV, however, during the experimental trials the laptop was used for simplicity and to enable more seamless algorithm development [Ladosz et al., 2019]. Furthermore, 
the data sent consisted only of a concentration measurement and 3D location coordinates. The Robot Operating System (ROS) framework was used for all communications between the autopilot and the on board computer, and likewise between the on-board computer and the ground-station.

\subsection{Contributions}

Gas source localisation has been an active area of academic research for some time. In spite of this, existing experimental results have rarely been obtained in realistic environments or even outdoors. To the best of the authors knowledge, gas source localisation using a UAV has only been achieved with a single system [Neumann et al., 2013]. This was a significant step forward, simultaneously extending previous work to an outdoor environment and utilising a UAV that could estimate the wind vector using its inertial measurement unit [Neumann and Bartholmai, 2015]. The experimental results were impressive, however, there were still some limitations at this stage: the search area was quite narrow and two dimensional, a fan at the source was used to create a nice flow to help spread the gas, the UAVs altitude was held manually, it was initiated from within the gas plume, and finally, the emission rate of the source was not estimated. All the former points are addressed in this paper.

Source term estimation is another popular area of research, with significant experimental results obtained using high quality datasets from experimental trials of tracer gas dispersion such as the Joint Urban 2003 study in Oklahoma [Allwine et al., 2004]. Contributions in this area typically focus on using a network of static gas detectors [Rao, 2007, Hutchinson et al., 2017b]. In the present study, a UAV is guided fully autonomously to collect the spatial temporal data required to estimate the parameters of a dispersive release. When using a static network, gas concentration samples are typically averaged over a period of a minute or more. Given the short flight time of a UAV this is greatly decreased, resulting in significantly different outputs from the sensor; characterised by greater intermittency, or non detections, and increased noise. A new likelihood function is used to handle the intermittent detections and greater uncertainty incurred by the shorter sampling periods. Furthermore, two simple, fast running dispersion models proposed in the literature for source term estimation are compared using the unique experimental data collected by the UAV. To the best of the authors knowledge, the experimental trials described in this paper mark the first occasion where a UAV is used, in realistic conditions, to search for and estimate the source term parameters of a gaseous release. This is a significant step towards an operational system.

The theoretical foundations of this paper were predominantly a result of earlier work that has been verified in simple simulation studies, datasets collected in a turbulent water channel, or by using a ground robot 
indoors [Hutchinson et al., 2018a]. In addition to the moderate adjustments to enable the algorithms practical implementation with a UAV, such as a novel likelihood function, the main contributions of this work are of a practical and experimental nature, as follows:

- A complete UAV based gas source estimation system has been developed consisting of gas sensors, a UAV, a ground control station, and a source estimation algorithm.

- The trials mark the first experimental result of source term estimation performed using gas measurements from an autonomous UAV.

- The source estimation performance is assessed with regards to the UAVs altitude, the distance between gas measurements and the wind speed or atmospheric stability.

- The experiments in general are rare, where a gas source is localised in an outdoor environment rather than in more controlled indoor arenas where fans are used to generate wind.

- Two well known models are compared using the unique experimental data.

Given such an immature area to obtain experimental results there were several observations and lessons learned during the outdoor trials. This has lead to new insights and subsequently, new areas identified for future research.

\subsection{Outline}

The remainder of the paper is outlined as follows. Firstly, to provide some background and to familiarise the reader with the research area, related work are reviewed in Section 2. In Section 3, the setup of the system is described in greater detail. A formal description of the problem is given in Section 4. In Section 5, the Bayesian estimation of the source parameters is described, including formulations of the models used and the computational implementation of the algorithm. Experimental trials are presented in Section6, including the setup, implementation remarks, an illustrative run and the results. Discussions and lessons learned are provided in Section 7, and finally, conclusions and ideas for future research are given in Section 8 .

\section{Related work}

Gas sensing using UAVs is a fairly new but rapidly growing area of research. It is quickly becoming more popular as a result of the improvements and reductions in the cost and size of gas detectors and UAVs. 
Applications of gas sensing UAVs, and research concerning the use and integration of gas detectors and aerial platforms are briefly reviewed in this section, followed by other related work on source localisation and source term estimation. The algorithms and system design taken forward in this paper are justified in comparison to the alternative methods.

\subsection{Gas sensing on UAVs}

The ability to sense a gas from a UAV has numerous proposed applications including: detecting gas leaks [Smith et al., 2016, Smith et al., 2017, Luo et al., 2018]; monitoring various sources of air pollution [Chang et al., 2016, Villa et al., 2016, Aurell et al., 2017, Alvarado et al., 2017]; measuring important variables in greenhouses [Roldán et al., 2015]; and exploring sources of methane emissions [Greatwood et al., 2017, Neumann et al., 2017]. The UAVs used in aerial gas sensing research include both fixed wing and rotary wing platforms [Alvarado et al., 2015]. Within the literature, research is typically focused on the application of the gas sensing UAVs and the data they can gather, rather than the post processing of such information which is the core of the present study.

One of the dominant factors to consider when measuring a gas using a UAV is the effect of the rotors on the dispersion of the gas and the output from the sensor. This effect has been taken into consideration in the past and research has been conducted to determine the optimal position of the gas detector and the effect on the sensor measurement [Roldán et al., 2015, Chang et al., 2016, Smith et al., 2016]. Some of the potential sensor positions proposed include: under the rotors of the UAV, in the centre of the platform raised above or below it, in the space between the UAV rotors, and extended on an arm away from the platform and its effect on the gas. Through computational fluid dynamics (CFD) studies, smoke visualisation experiments, and pressure and airflow measurements around the UAV, some conclusions can be drawn, despite conflicting results. The general consensus is that the effect of the rotors is to decrease the measurement from the gas detector and increase its uncertainty [Villa et al., 2016]. The most accurate measurements would come from a sensor outside of the disturbed region of airflow, however, this would be more likely to cause stability issues whilst in flight. A pumped system could be implemented on the vehicle, where the inlet would be away from the platform, still, this would add undesirable weight to the system. For these reasons, the most common placement seen in the literature is in a raised position, in the centre of the platform [Roldán et al., 2015, Alvarado et al., 2017]. The focus of the present work is on validating a source term estimation algorithm using a UAV, consequently, the effect of the rotors has not been prioritised. Nevertheless, the effect on the source estimates is discussed in the results. Given the huge increase in applications and experiments 
involving gas sensing on UAVs, it is envisaged that bespoke new sensors, designed for UAVs will have a great benefit and will be an important area for future research.

\subsection{Source localisation}

The goal of source localisation is closely aligned with this present work since the aim is to guide a sensor equipped, mobile platform to the source of a gas or odour to establish its origin. The methods differ because source localisation approaches typically do not estimate other parameters of the source term. Despite providing less information about the release, an advantage of such an approach is the potential alleviation of the requirement for a dispersion model fit for the scenario at hand. The methods therefore, can range from simple rule based reactive approaches to more complex probabilistic methods [Voges et al., 2014]. Source localisation has received substantial interest lately with the development of several robotic platforms and algorithms. A survey on odour source localisation using a mobile robot is given in [Kowadlo and Russell, 2008] and a detailed review on chemical sensing robots with more focus on experimental studies and practical application is given in [Ishida et al., 2012]. The majority of the experiments are performed in indoor environments with artificial wind produced by fans. Some notable examples of outdoor experiments include [Li et al., 2011] and [Neumann et al., 2013], where particle filter based algorithms are used to estimate the source location using an unmanned ground or aerial vehicle.

An interesting approach to guide a robot to a gas source was suggested in [Vergassola et al., 2007], where an information based reward was formulated. This new method received considerable interest in the area and has lead numerous researchers to propose modifications and extensions to the algorithm [Moraud and Martinez, 2010, Ristic et al., 2016, Masson, 2013]. Experimental studies of such an approach have so far been limited to indoor areas with artificially generated wind [Voges et al., 2014].

An alternative approach to source localisation does not attempt to direct a robot, or searcher, to the source of the emission. Rather, this technique capitalises on an interesting observation on sensing characteristics in plumes; where variance and fluctuations in gas concentration tend to be greater nearer the source [Hernandez Bennetts et al., 2012]. This feature has been used to simultaneously estimate the source position whilst mapping the gas distribution. Methods proposed include the kernel DM+V algorithm, which uses in-situ point measurements to map the concentration mean and variance [Lilienthal et al., 2009, Hernandez Bennetts et al., 2012], and a gas tomography algorithm which uses integral measurements from a tunable diode laser absorption spectroscopy (TDLAS) sensor to map the mean and fluctuations in concentration [Bennetts et al., 2014]. The results of experimental trials conducted in different indoor and outdoor environments verified 
the approach using data collected during sweep patterns performed using aerial and ground based robots and various sources [Hernandez Bennetts et al., 2012].

\subsection{Source term estimation}

Contrary to source localisation, the goal of source term estimation (STE) is to estimate several parameters of the source, including its location, in order to learn more about the origin of the release [Hutchinson et al., 2017b]. This can provide more useful information, however, it can require more computational power in order to run ATD models and optimisation or Bayesian inference algorithms than the more simple rule based localisation methods. STE is most commonly performed using a network of static gas detectors and meteorological stations [Rao, 2007, Redwood, 2011, Singh et al., 2015, Hutchinson et al., 2017b]. Accidents such as the Fukushima nuclear disaster can benefit greatly from such a technique because a network of static sensors are put in place around areas of high risk, such as a nuclear plant, to monitor for such an event [Chino et al., 2011, Winiarek et al., 2014]. On the other hand, it is infeasible to cover all areas with a network of static detectors.

A critical component of STE algorithms is the underlying ATD model. For applications that do not require a rapid response, it would be reasonable to use more complex and potentially more accurate methods such as CFD to model the expected observations from the detectors. When a rapid response is required, more simple models may be more appropriate, which can be derived using analytical solutions to the advection diffusion equations with various assumptions. In this work, two models are compared using the unique data collected during the experimental trials: The Gaussian plume equation [Wang et al., 2017] and an Isotropic plume equation [Vergassola et al., 2007]. The predominant difference between the models lies in the specification of the diffusivity parameters.

The robots used to perform source localisation can similarly be used for STE by replacing the data collected by the static network. This leads to the problem of how to guide the platform to collect enough useful spatial temporal data to effectively estimate the parameters of the release. The most intuitive approaches would be to continue the trajectory of a typical gas source localisation algorithm, or to perform a systematic search pattern such as a parallel sweep or an Archimedean spiral [Champagne et al., 2003, Hutchinson et al., 2017a]. Alternatively, information based guidance methods have been proposed to guide the robot to the expected most informative locations to take measurements. These approaches have been proposed to estimate the source terms of radiological releases [Ristic et al., 2010], a diffusive release [Ristic et al., 2016,Hutchinson et al., 2018b] and to complement an existing static network of detectors [Šmídl and Hofman, 


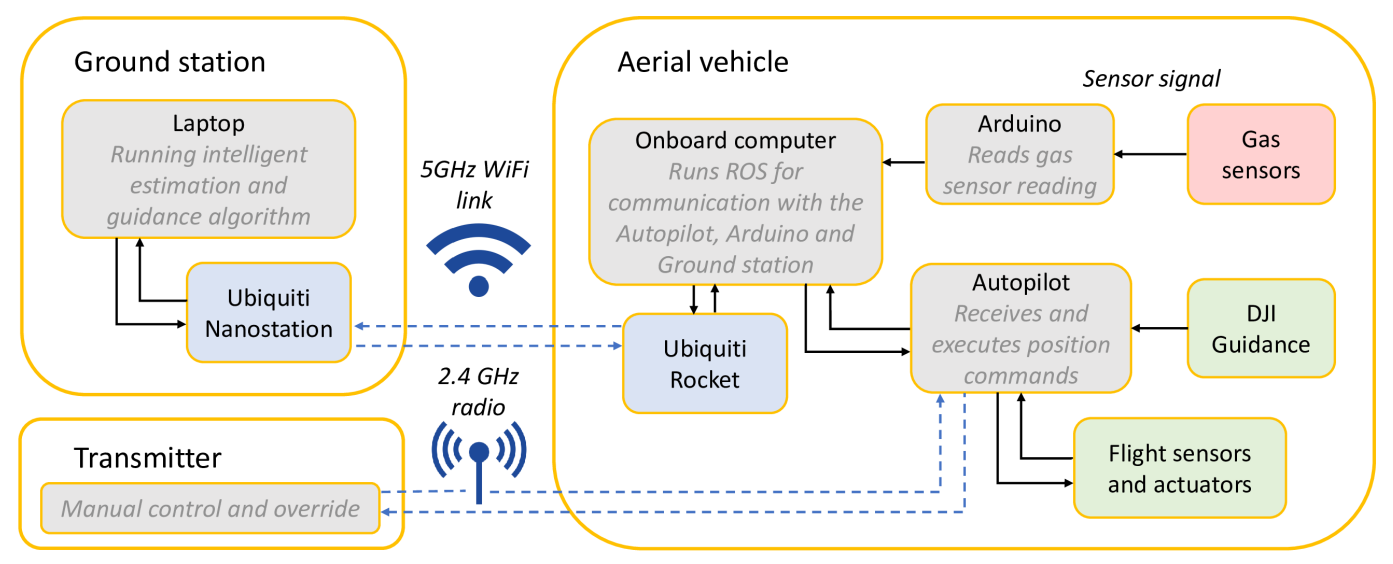

Figure 2: System overview: The measurements from the PID gas detector are read by an Arduino Uno. The ground station and the on-board computer communicate over $5 \mathrm{GHz}$ WiFi. The Arduino and autopilot communicate with the on-board computer via serial and UART connections. The transmitter is used to switch between manual and autonomous flight. The DJI Guidance system is connected to the autopilot to provide more accurate localisation in flight.

2013]. In [Hutchinson et al., 2018a], an information based STE method was implemented on a ground robot and assessed in indoor experiments where fans were used to simulate a wind field. This result motivates extension of the Bayesian estimation approach to handle real outdoor environments and deployment on a aerial platform. The use of multiple robots has also been proposed, employing an uncertainty driven exploration strategy to plan their path whilst estimating the locations and intensities of multiple sources [Wiedemann et al., 2017]. The method was tested with real robots in hardware in the loop simulations using simulated gas sensor data.

Despite several proposals to guide a mobile platform to estimate the source term of a release, real experimental results of STE using a UAV, in outdoor conditions, have not previously been produced. The most closely related experimental result used a manned aircraft equipped with a highly sensitive methane detector. Observations during sweep search patterns were used to estimate the source terms of methane releases from landfill sites using a reversible jump Markov chain Monte Carlo algorithm [Hirst et al., 2013]. Filling this gap, by performing STE using an autonomous UAV, is a key contribution of the present study.

\section{System overview}

The main components of the system are a quadrotor UAV platform, an on-board computer, a ground station laptop and the gas sensing payload. An overview of the system is outlined in Fig 2 and a photo of the equipment prior to an experimental trial is shown in Fig 3. The remainder of this section shall further 


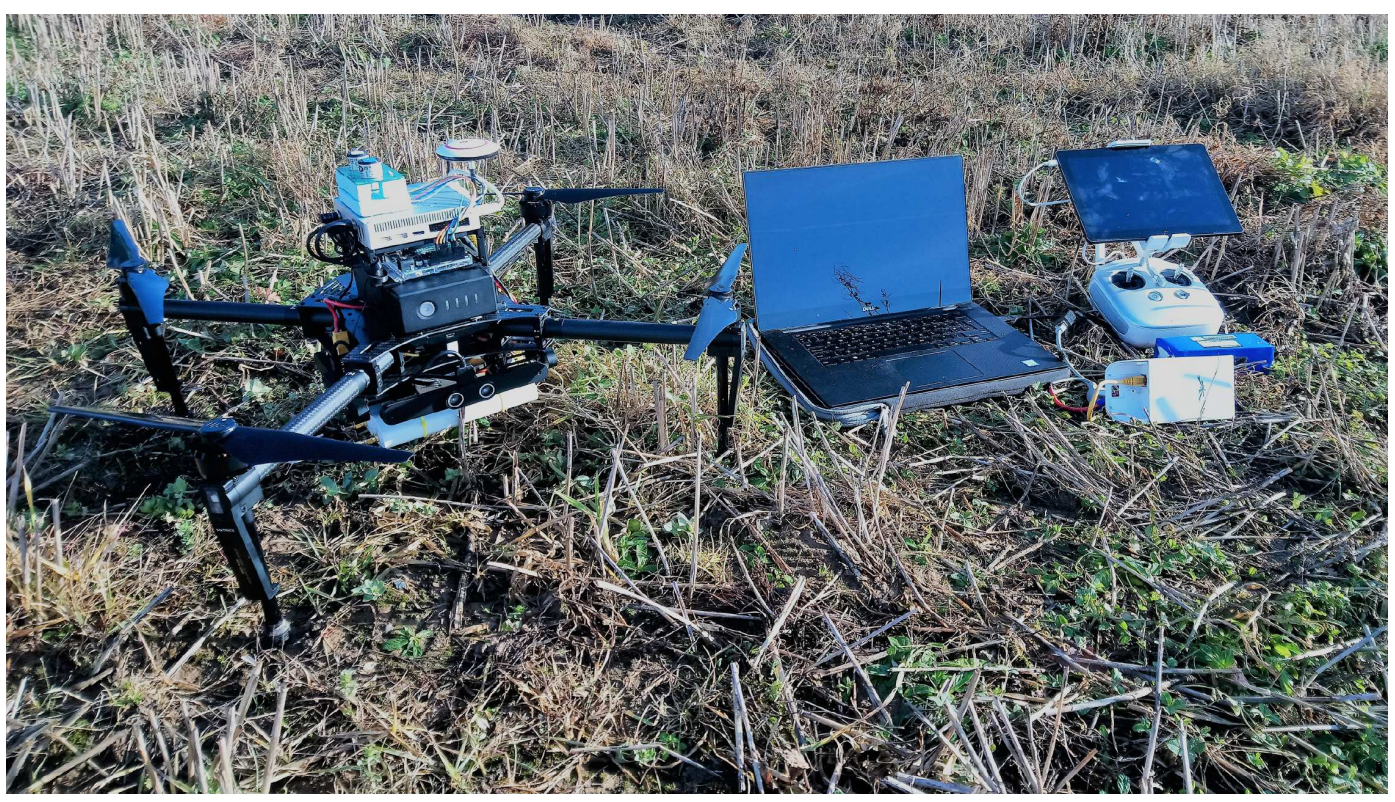

Figure 3: System components: DJI Matrice 100 UAV, ground station laptop, WiFi equipment and radio transmitter.

describe the system components and its set-up.

\subsection{Aerial platform}

The aerial platform is a DJI Matrice 100 as shown in Fig 3. Among other components, it is equipped with: DJI's guidance system, to support accurate localisation; an autopilot; a GPS; and DJI's on-board computer called the manifold. The manifold is a reasonably powerful computer based off an Nvidia Tegra. Although, in the present study, the algorithms are run on the ground station laptop, in the future they could be deployed to run on-board the UAV. This would have great benefits in large or cluttered scenarios, such as urban areas, where wireless communication could become unstable. The on-board computer communicates with the autopilot via UART and with the ground station via $5 \mathrm{GHz} \mathrm{WiFi}$. The WiFi communication is achieved via a bridge between a Ubiquiti Rocket on-board the Matrice 100 and a Ubiquiti M5 Nanostation which is connected to the laptop on the ground. A $5 \mathrm{GHz}$ link is selected over the longer range $2.4 \mathrm{GHz}$ to avoid interference with the remote controller. The $\mathrm{ROS}^{1}$ software framework is used for communication among the system components. The dji_sdk ${ }^{2}$ package facilitates communication between the on-board computer and the autopilot. Additionally, the data from the gas sensor is read by an Arduino Uno and input to the ROS network using the rosserial_arduino ${ }^{3}$ package. The ROS network allows the autopilot data, sensor data, and

\footnotetext{
${ }^{1}$ http://www.ros.org

${ }^{2}$ http://wiki.ros.org/dji_sdk

${ }^{3}$ http://wiki.ros.org/rosserial_arduino
} 


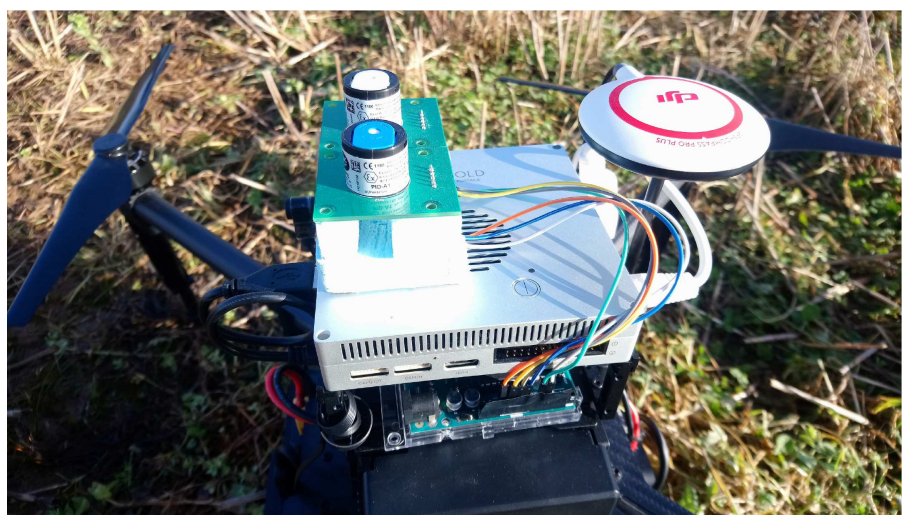

Figure 4: PID gas sensors connected to an Arduino Uno on-board the UAV.

commands from the ground station to be shared among the connected components in the system.

The set-up of the system was motivated by the ability to quickly test algorithms developed in Matlab without the requirement to compile the code on the UAV. This enabled rapid development and adjustments to the algorithm whilst out in the field. During the experiments, the 3D position of the UAV and a sensor reading are sent from the on-board computer to the ground station. The ground station updates its estimates of the source term parameters using a sequential Bayesian algorithm, and sends a new position demand to the aerial vehicles on-board computer. The dji_sdk package performs the lower level control to manoeuvre the UAV to the new position.

\subsection{Gas sensing payload}

The UAV is equipped with a PID gas detector to take measurements of the hazardous gas, as shown in Fig 4. The PID sensors were chosen as they are a reasonable price and highly sensitive to a large number of chemicals. The output of the PID detector is a reading related to the concentration of a standard volatile organic compound (VOC), isobutelene. A data sheet is provided with the sensors to approximate this to a parts per billion (ppb) measurement of the target gas, which is subsequently converted to a concentration measurement in $g / m^{3}$. As shown in Fig 4, the sensor is wired to an Arduino Uno ${ }^{4}$, which communicates with and draws power from the on-board computer via a serial connection.

As discussed in Section 2, the literature on the optimal placement of gas sensors on UAVs is conflicting. Some researchers report that near to the propellers is better for sensing, whilst others observe the opposite and suggest they should be kept out of the induced airflow. The most common suggestion is in an elevated

\footnotetext{
${ }^{4}$ https://www.arduino.cc/
} 
position in the middle of the UAV, which is adopted in this work as shown in Fig 4. Although this is likely to effect the reading from the sensor, it is structurally more favourable than extending it on an arm, more lightweight than using a pumped system, and it will protect the sensor in the event of a hard landing. Sensor placement, and possibly correction factors to account for the UAVs rotor effect are left as an area for future research. However, the effect on the source estimation results are examined and discussed in Sections 6 and 7.

\section{Problem description}

After an event such as an earthquake or explosion, a large area of concern exists where there is the potential for a hazardous release from damaged pipes or chemical facilities. In response to a suspicious smell, it could be challenging to find the source or determine if it is hazardous. After an act of terrorism involving gas, it would be of paramount importance to locate the source without endangering the lives of responders. During an important event it may be desirable to monitor the surrounding area for signs of a dangerous release. Given such an area of interest, the goal of this work is to provide an algorithm to autonomously search for and estimate the parameters of a release, with a high degree of accuracy and in a short amount of time.

The zone of interest, parameterised by the three dimensional volume $\Omega \subset \mathbb{R}^{3}$, will be used to initialise the search area of the algorithm. This could be the region where a suspicious odour is reported, a region of interest to survey, an area along a pipeline or the area around a chemical facility. The UAV, equipped with the relevant gas detector payload, is to navigate within the area to estimate the release parameters otherwise known as the source term. This shall provide responders with information about the location of the release, as well as the necessary inputs to an ATD model to produce a forecast of the hazard.

The UAV is aware of its location $\mathbf{p}_{k}=\left[\begin{array}{lll}x_{k} & y_{k} & z_{k}\end{array}\right]^{\top} \in \Omega$ within the domain. In this present study, this is achieved via fusion of GPS, IMU, ultrasonic and stereo image data. The gas detector on-board the UAV observes point-wise measurements of the gas concentration $z_{k} \in \mathbb{R}^{+}$. The meteorological parameters are provided by a local weather station. The location stamped measurements and meteorological observations are used to estimate the parameters of the source $\Theta_{k}$, which in this work, is given by:

- Cartesian coordinates of the source $\mathbf{p}_{s}=\left[\begin{array}{lll}x_{s} & y_{s} & z_{s}\end{array}\right]^{\top} \in \Omega$ in meters $(m)$.

- Emission rate/strength of the source $q_{s} \in \mathbb{R}^{+}$in grams per second $(g / s)$.

- The wind speed $u_{s} \in \mathbb{R}^{+}$in meters per second $(\mathrm{m} / \mathrm{s})$ and direction $\phi_{s} \in \mathbb{R}$ in radians $(\mathrm{rad})$. 
- Model dependant diffusion parameters $\zeta_{s}=\left[\begin{array}{ll}\zeta_{s 1} & \zeta_{s 2}\end{array}\right]^{\top} \in \mathbb{R}^{+}$which relate to the spread of the gas concentration from the source.

Hence, the parameter vector of the source term can be defined as:

$$
\Theta_{k}=\left[\begin{array}{lllll}
\mathbf{p}_{s}^{\top} & q_{s} & u_{s} & \phi_{s} & \zeta_{s}
\end{array}\right]^{\top}
$$

The key parameters of the source term are its location and emission rate. The remaining parameters are incorporated to add robustness to the system and account for uncertainties.

The UAV is to autonomously search the environment, collecting point observations $\mathbf{z}_{1: k}=\left\{z_{1}, \ldots, z_{k}\right\}$ from the gas detector at discrete time steps $k=1, \ldots, k$ and at known locations $\mathbf{p}_{1: k}=\left\{\mathbf{p}_{1}, \ldots, \mathbf{p}_{k}\right\}$. At each time step $k$, the estimates of the source parameters $\Theta_{k}$ are updated by drawing the inference on the probabilistic distribution $p\left(\Theta_{k} \mid \mathbf{z}_{1: k}\right)$. The next location to make an observation with the gas detector $\mathbf{p}_{k+1}$ is then selected, and navigated towards, to begin the next iteration of the algorithm.

\section{Source term estimation}

In such a scenario where input variables, measurements and underlying models are fraught with uncertainty, a probabilistic approach is preferable so that the errors can be accounted for by designing a likelihood function to reflect such uncertain conditions. Under this approach, the uncertainty in the source term estimates can be captured within a probability density function (pdf). Bayes' theorem is used to update the estimates of the source parameter vector $\Theta_{k}$ in a recursive manner given the measurements from the gas detector $\mathbf{z}_{1: k}$ and prior information.

Using the Bayesian framework, the current state of knowledge regarding the source parameters is represented by a posterior probability distribution $p\left(\Theta_{k} \mid \mathbf{z}_{1: k}\right)$, where $\mathbf{z}_{1: k}$ implies that the measurement data are collected at locations $\mathbf{p}_{1: k}$, respectively. In response to new measurement data from the gas detector $z_{k+1}$, the posterior distribution is updated according to Bayes' rule, such that

$$
p\left(\Theta_{k+1} \mid \mathbf{z}_{1: k+1}\right)=\frac{p\left(z_{k+1} \mid \Theta_{k+1}\right) p\left(\Theta_{k+1} \mid \mathbf{z}_{1: k}\right)}{p\left(z_{k+1} \mid \mathbf{z}_{1: k}\right)}
$$


where

$$
p\left(z_{k+1} \mid \mathbf{z}_{1: k}\right)=\int p\left(z_{k+1} \mid \Theta_{k+1}\right) p\left(\Theta_{k+1} \mid \mathbf{z}_{1: k}\right) \mathrm{d} \Theta_{k+1} .
$$

The initial prior distributions $\pi\left(\Theta_{0}\right) \equiv p\left(\Theta_{0}\right)$ of the source parameters are assumed to be provided to the algorithm, these can be obtained autonomously through sensory data or by user input. If information concerning the source term is available prior to the search, it can be exploited through an appropriate distribution to represent the prior knowledge known about the release. In the absence of information, the prior can be set to an uninformative distribution. For example, the prior distribution for the location of the source is a uniform distribution that is bounded by the domain $\Omega$. In subsequent iterations, the prior distributions are replaced by the posteriors to reflect the information gained from the previous sequence. In this Bayesian inferential framework, it is also assumed that the source term is constant, i.e. $\Theta_{k+1}=\Theta_{k}$, which implies $p\left(\Theta_{k+1} \mid \mathbf{z}_{1: k}\right)=p\left(\Theta_{k} \mid \mathbf{z}_{1: k}\right)$.

\subsection{The likelihood function}

To construct the likelihood function $p\left(z_{k+1} \mid \Theta_{k+1}\right)$ used in Eq. (2), there must be a method of linking sensor measurements $z_{k}$ with the expected observations. To do this, a model of the dispersion from a source and a model of the sensor response are required.

\subsubsection{Dispersion models}

The dispersion model will provide the expected concentration at position $\mathbf{p}_{k}$ produced from a hypothesised source with parameters $\Theta_{k}$, given as $\mathcal{M}\left(\mathbf{p}_{k}, \Theta_{k}\right)$. Any relevant model can be used; there exist highly complex particle tracking models, CFD techniques, or equations derived from analytical solutions to the advectiondiffusion equations such as the Gaussian plume dispersion model. The model is interchangeable without any other changes to the algorithm, and should be chosen to reflect the current scenario. For example, the NAME dispersion model is used by the UK Met Office to forecast long range ash dispersion from a volcanic eruption [Jones et al., 2007], whereas CFD based methods have been developed for complex geometries such as urban environments [Keats et al., 2007,Efthimiou et al., 2017]. In this work, two models are compared, both derived from analytical solutions to the advection diffusion equation with various assumptions: The standard Gaussian plume (GP) model [Wang et al., 2017], and a more simplified model assuming isotropic diffusion [Vergassola et al., 2007] which shall be referred to as the Isotropic plume (IP) model. Both models are fast running and based on the assumption of a steady state plume with a consistent mean wind velocity, source strength, and turbulent conditions. The principle difference among the methods is in the specification 
of the diffusion parameters $\zeta_{\mathbf{s}}=\left[\zeta_{s 1}, \zeta_{s 2}\right]$ and the assumptions therein.

Gaussian plume model The GP model approximates the spread of the gas from the source in the crosswind, horizontal and vertical directions using measurements or approximations of atmospheric stability. To account for uncertainties, the diffusion parameters are adopted from [Senocak et al., 2008], resulting in $\left[\zeta_{s 1}, \zeta_{s 2}\right]$ representing stochastic diffusion terms in the horizontal and vertical directions. Subsequently, the expected concentration to be read by a detector at position $\mathbf{p}_{k}$ from a source with parameters $\Theta_{k}$ using the Gaussian plume model is given as

$$
\mathcal{M}\left(\mathbf{p}_{k}, \Theta_{k}\right)=\frac{q_{s}}{u_{s} \sigma_{y, k} \sigma_{z, k} 2 \pi} \exp \left(\frac{-c_{k}^{2}}{2 \sigma_{y, k}^{2}}\right) \times\left[\exp \left(\frac{-\left(z_{k}-z_{s}\right)^{2}}{2 \sigma_{z, k}^{2}}\right)+\exp \left(\frac{-\left(z_{k}+z_{s}\right)^{2}}{2 \sigma_{z, k}^{2}}\right)\right],
$$

where $c_{k}$ is the crosswind distance from the source, and, given that the downwind distance from the source is $d_{k}$, the standard deviations of concentration in the crosswind and vertical directions are:

$$
\sigma_{y, k}=\frac{\zeta_{s 1} d_{k}}{\sqrt{\left(1+0.0001 d_{k}\right)}} \quad \text { and } \quad \sigma_{z, k}=\frac{\zeta_{s 2} d_{k}}{\sqrt{\left(1+0.0001 d_{k}\right)}}
$$

Isotropic plume model The Isotropic model assumes isotropic diffusion from the source. Following [Vergassola et al., 2007], the diffusion terms $\left[\zeta_{s 1}, \zeta_{s 2}\right]$ represent the diffusivity of the gas in the environment, and the average lifetime of the gas. Given the model, the expected concentration to be read by a detector at position $\mathbf{p}_{k}$ from a source at position $\mathbf{p}_{s}$, releasing gas at a rate of $q_{s}$ with average lifetime $\zeta_{s 2}$ in an environment with mean wind speed $u_{s}$, wind direction $\phi_{s}$ and diffusivity $\zeta_{s 1}$ is given by:

$$
\begin{aligned}
& \mathcal{M}\left(\mathbf{p}_{k}, \Theta_{k}\right)=\frac{q_{s}}{4 \pi \zeta_{s 1}\left\|\mathbf{p}_{k}-\mathbf{p}_{s}\right\|} \exp \left[\frac{-\left\|\mathbf{p}_{k}-\mathbf{p}_{s}\right\|}{\lambda}\right] \times \\
& \exp \left[\frac{-\left(x_{k}-x_{s}\right) u_{s} \cos \phi_{s}}{2 \zeta_{s 1}}\right] \exp \left[\frac{-\left(y_{k}-y_{s}\right) u_{s} \sin \phi_{s}}{2 \zeta_{s 1}}\right],
\end{aligned}
$$

where

$$
\lambda=\sqrt{\frac{\zeta_{s 1} \zeta_{s 2}}{1+\left(u_{s}^{2} \zeta_{s 2}\right) /\left(4 \zeta_{s 1}\right)}}
$$

An example plot from each of the models is shown in Fig 5, where the sensor model to be described in the next section has been applied. The main difference in the outputs of the models is seen in the vicinity of the 


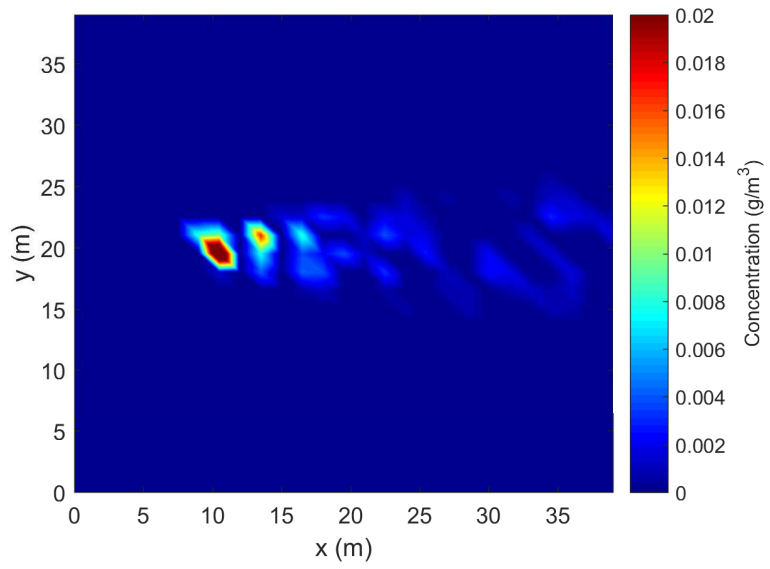

(a) Gaussian plume model

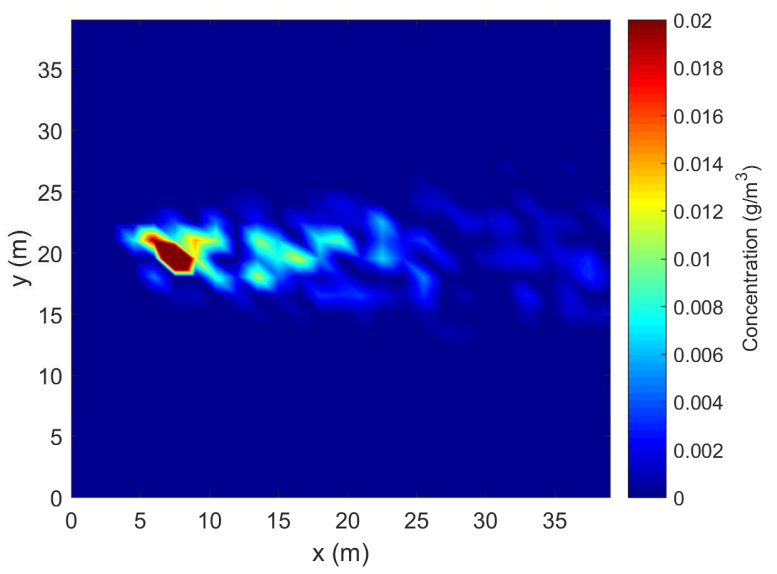

(b) Isometric plume model

Figure 5: Example plots of the expected observations $z_{k}$ of the UAV flying at a $2.5 \mathrm{~m}$ altitude using: (a) the GP model; and (b) the IP model. The source had parameters: $x_{s}=4, y_{s}=20, z_{s}=1.5, q_{s}=1, u_{s}=5$, and $\phi_{s}=90^{\circ}$.

source, particularly upwind. Upwind of the source, the GP model (Fig 5a) assumes zero mean concentration from the source whereas the IP model (Fig 5b) does not. The GP model is more popular in the literature, it is extensively studied, and even accepted commercially. However, the model is typically used on a larger scale than the experiments conducted in this paper and as depicted in the example figures.

\subsubsection{Gas sensing model}

To form the likelihood function used in Eq. (2), the measurements from the gas detector must be related to the expected observations deduced from the dispersion models. The measurement data $\mathbf{z}_{1: k}$ features detection events, where measurements from the gas detector picked up some concentrations from the source, and non detection events, where the measurement did not surpass a pre-specified threshold $z_{t h r}$. The threshold is set high enough to minimise false detections, whilst maintaining sufficient sensitivity. The observational data, and subsequently the likelihood function, can be split among these detections $\bar{z}_{k}$ and non detections $\underline{z}_{k}$ [Yee, 2017] as:

$$
p\left(z_{k} \mid \Theta_{k}\right)= \begin{cases}p\left(\bar{z}_{k} \mid \Theta_{k}\right) & \text { if } z_{k}>z_{t h r} \\ p\left(\underline{z}_{k} \mid \Theta_{k}\right) & \text { if } z_{k} \leq z_{t h r}\end{cases}
$$

The observational model linking detection data $\bar{z}_{k}$ with the source term parameters $\Theta_{k}$ is given as 


$$
\bar{z}_{k}=\mathcal{M}\left(\mathbf{p}_{k}, \Theta_{k}\right)+\bar{v}_{k},
$$

where $\bar{v}_{k}$ encapsulates the various errors between the measured and modelled concentration at a particular position $\mathbf{p}_{k}$. The discrepancy can arise from measurement error, input error, model error and stochastic uncertainty [Rao, 2005]. Given the limited knowledge of the errors between predicted and measured concentrations, application of the maximum entropy principle suggests the Gaussian distribution as the most conservative choice for the likelihood function [Jaynes, 2003, Yee, 2017]. Thus the likelihood function for a detection event is as follows:

$$
p\left(\bar{z}_{k} \mid \Theta_{k}\right)=\frac{1}{\sigma_{k} \sqrt{2 \pi}} \exp \left[-\frac{\left(\bar{z}_{k}-\mathcal{M}\left(\mathbf{p}_{k}, \Theta_{k}\right)\right)^{2}}{2 \sigma_{k}^{2}}\right],
$$

where the variance $\sigma_{k}$ is a function of the modelled concentration such that $\sigma_{k} \propto \mathcal{M}\left(\mathbf{p}_{k}, \Theta_{k}\right)$.

A non detection event on the other hand, can be caused by three hypothesised scenarios: The concentration measurement is only a result of background and instrument noise $\mathcal{E}_{b}$; the non detection is a result of intermittency caused by turbulence or a missed detection $\mathcal{E}_{m}$, typically exacerbated by the short sampling intervals of the UAV; or, the concentration includes contributions from both the source and background, although it did not amount to a value above the concentration threshold $\mathcal{E}_{s}$. Combining the three hypotheses results in the following likelihood of a non detection:

$$
p\left(\underline{z}_{k} \mid \Theta_{k}\right)=p\left(\mathcal{E}_{b}\right) \cdot p\left(\underline{z}_{k} \mid \mathcal{E}_{b}, \Theta_{k}\right)+p\left(\mathcal{E}_{m}\right) \cdot p\left(\underline{z}_{k} \mid \mathcal{E}_{m}, \Theta_{k}\right)+p\left(\mathcal{E}_{s}\right) \cdot p\left(\underline{z}_{k} \mid \mathcal{E}_{s}, \Theta_{k}\right)
$$

where the probability of each event is given as $p\left(\mathcal{E}_{b}\right)=P_{b}, p\left(\mathcal{E}_{m}\right)=P_{m}$ and $p\left(\mathcal{E}_{s}\right)=P_{s}$ and $P_{b}+P_{m}+P_{s}=1$. Assuming the background noise and contributions from the source can be modelled as normal distributions the likelihood function for a non detection can be written as

$$
p\left(\underline{z}_{k} \mid \Theta_{k}\right)=\left(P_{b} \times \frac{1}{2}\left[1+\operatorname{erf}\left(\frac{z_{t h r}-\mu_{b}}{\sigma_{b} \sqrt{2}}\right)\right]\right)+P_{m}+\left(P_{s} \times \frac{1}{2}\left[1+\operatorname{erf}\left(\frac{z_{t h r}-\left(\mu_{b}+\mathcal{M}\left(\mathbf{p}_{k}, \Theta_{k}\right)\right)}{\sigma_{k} \sqrt{2}}\right)\right]\right)
$$

where $\mu_{b}$ and $\sigma_{b}$ are the mean and variance of the background noise and erf() denotes the error function. The values of $P_{b}, P_{m}$ and $P_{s}$ were set during the experiments. 
Given the appropriate models and Bayesian formulations, the next section will describe a method to implement the probabilistic estimation of the source parameters.

\subsection{Sequential Bayesian implementation}

The Bayesian estimation of the source parameters is implemented in the sequential Monte Carlo framework using a particle filter. The output is an approximation of the posterior distribution $p\left(\Theta_{k} \mid \mathbf{z}_{1: k}\right)$, which represents the current state of knowledge about the source parameters. The posterior distribution from Eq. (2) is approximated by a set of weighted random samples $\left\{\Theta_{k}^{(i)}, w_{k}^{(i)}\right\}_{i=1}^{N}$, where $\Theta_{k}^{(i)}=$ $\left[\begin{array}{llllllll}x_{s, k}^{(i)} & y_{s, k}^{(i)} & z_{s, k}^{(i)} & q_{s, k}^{(i)} & u_{s, k}^{(i)} & \phi_{s, k}^{(i)} & \zeta_{s 1, k}^{(i)} & \zeta_{s 2, k}^{(i)}\end{array}\right]^{\top}$ is a sample representing a potential source term and $w_{k}^{(i)}$ is the corresponding normalised weighting such that $\sum_{i=1}^{N} w_{k}^{(i)}=1$. Given the weighted samples, the posterior distribution is approximated as:

$$
p\left(\Theta_{k} \mid \mathbf{z}_{1: k}\right) \approx \sum_{i=1}^{N} w_{k}^{(i)} \delta\left(\Theta_{k}-\Theta_{k}^{(i)}\right),
$$

where $\delta(\cdot)$ is the Dirac delta function. The sample weights are updated in a recursive manner by sequential importance sampling [Ristic et al., 2004]. At each time step, a set of new samples $\left\{\Theta_{k+1}^{(i)}\right\}_{i=1}^{N}$ can be drawn from a proposal distribution $q\left(\Theta_{k+1}^{(i)}\right)$, which should resemble the distribution $p\left(\Theta_{k+1} \mid \mathbf{z}_{1: k+1}\right)$. The corresponding un-normalised weights are then updated according to:

$$
\bar{w}_{k+1}^{(i)} \propto w_{k}^{(i)} \cdot \frac{p\left(z_{k+1} \mid \Theta_{k+1}^{(i)}\right) p\left(\Theta_{k+1}^{(i)} \mid \Theta_{k}^{(i)}\right)}{q\left(\Theta_{k+1}^{(i)} \mid \Theta_{k}^{(i)}, \mathbf{z}_{1: k+1}\right)} .
$$

The proposal distribution is typically used to update the samples to the next time step for estimating dynamic states. By assuming a time-invariant source term (i.e. the source position is fixed and the release rate is constant), the proposal distribution can be assumed to be identical to the posterior at time $k$. This leads to a simple algorithm where $\Theta_{k+1}^{(i)}=\Theta_{k}^{(i)}$ for $i=1, \ldots, N$ [Ristic et al., 2016]. Due to cancellation of terms in Eq. (14), the un-normalised particle weights are updated using the likelihood function and the previous weight as follows:

$$
\bar{w}_{k+1}^{(i)}=w_{k}^{(i)} \cdot p\left(z_{k+1} \mid \Theta_{k+1}^{(i)}\right) .
$$

The sample weights are then normalised as $w_{k+1}^{(i)}=\bar{w}_{k+1}^{(i)} / \sum_{i=1}^{N} \bar{w}_{k+1}^{(i)}$ to obtain the new approximation of the posterior.

Importance sampling is carried out sequentially at each time step. This can eventually lead to only a few 
particles with non-negligible weights, known as the degeneracy problem. To avoid sample degeneracy, the number of effective samples are estimated by:

$$
N_{e f f}=\frac{1}{\sum_{i=1}^{N}\left(w_{k}^{(i)}\right)^{2}}
$$

When the number of effective point estimates $N_{\text {eff }}$ falls below a pre-specified threshold $\eta$ the sample points are re-sampled. This can lead to another problem where highly weighted particles will be multiplied many times, leading to a lack of diversity. This problem is referred to as sample impoverishment. To improve the diversity of the random samples, the re-sampled estimates are regularised by drawing new samples from a Gaussian kernel. The new samples undergo an MCMC move step [Ristic et al., 2004], where they will be accepted with a probability proportional to their likelihood.

\section{$6 \quad$ Experimental trials}

In this section the experiments used to verify the system are described and the results are presented and discussed. Firstly, the experiment setup is outlined including information about the environment, the equipment used, the inputs to the algorithm and remarks on its implementation. Given the setup, an illustrative run of one of the trials is provided to further illustrate the experimental procedure and the capabilities of the algorithm. The results of all the conducted experimental trials are then summarised. Finally, the output estimates of the algorithm are assessed with regards to the measurement altitude of the UAV, the step increment in the sweep pattern, and the wind speed or atmospheric stability.

\subsection{Experiment setup}

The experiments were conducted outdoors in an open field, in order to verify the algorithm for the first time in real world atmospheric conditions, outside of simulation. Acetone was released into the atmosphere using a source comprising of ultrasonic diffusers and an air pump, as depicted in Fig 6 . The release rate of the source was obtained by measuring directly, the change in weight at the beginning and the end of the experiments and assuming that it was emitted at a constant rate. The release rate was typically $1.5 \mathrm{~g} / \mathrm{s}$, however, this would vary depending on atmospheric conditions such as temperature and pressure.

The field used during the experiments was located nearby Loughborough University, Leicestershire, UK. A large square within the field, containing the release, would represent the domain $\Omega$ which forms a part of the 
input to the algorithm. A photo of the environment is shown in Fig 7, featuring examples of the starting location of the UAV, the wind direction and the position of the source.

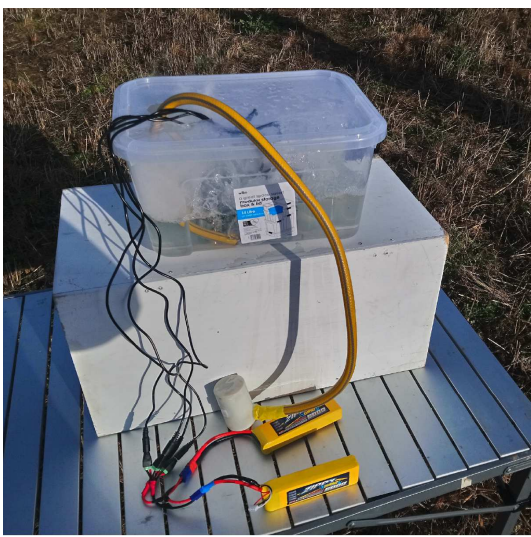

(a)

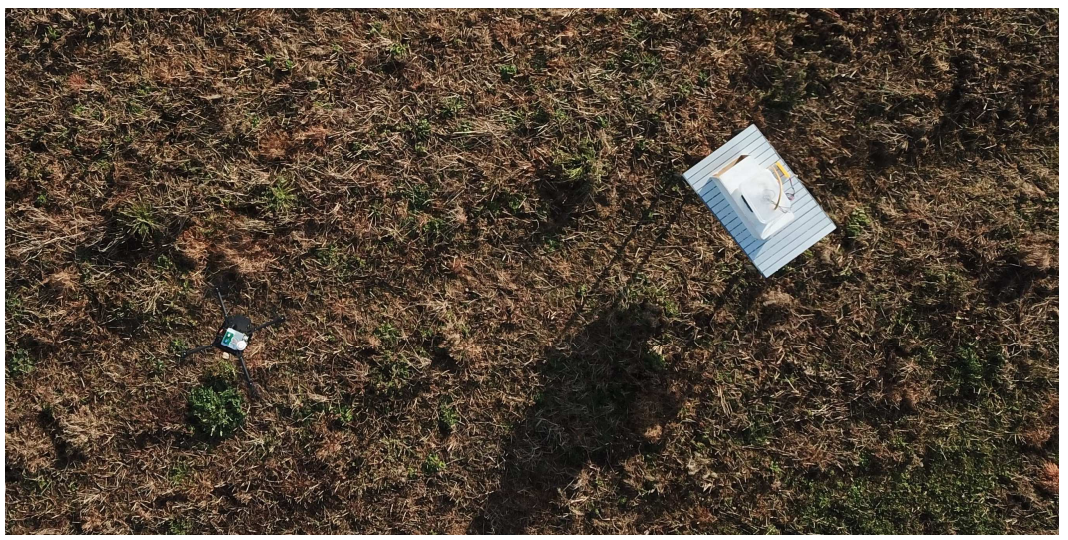

(b)

Figure 6: (a) Source set-up for the experiments comprising of acetone, diffusers and an air pump. (b) A snapshot of the source and UAV during an experimental trial.

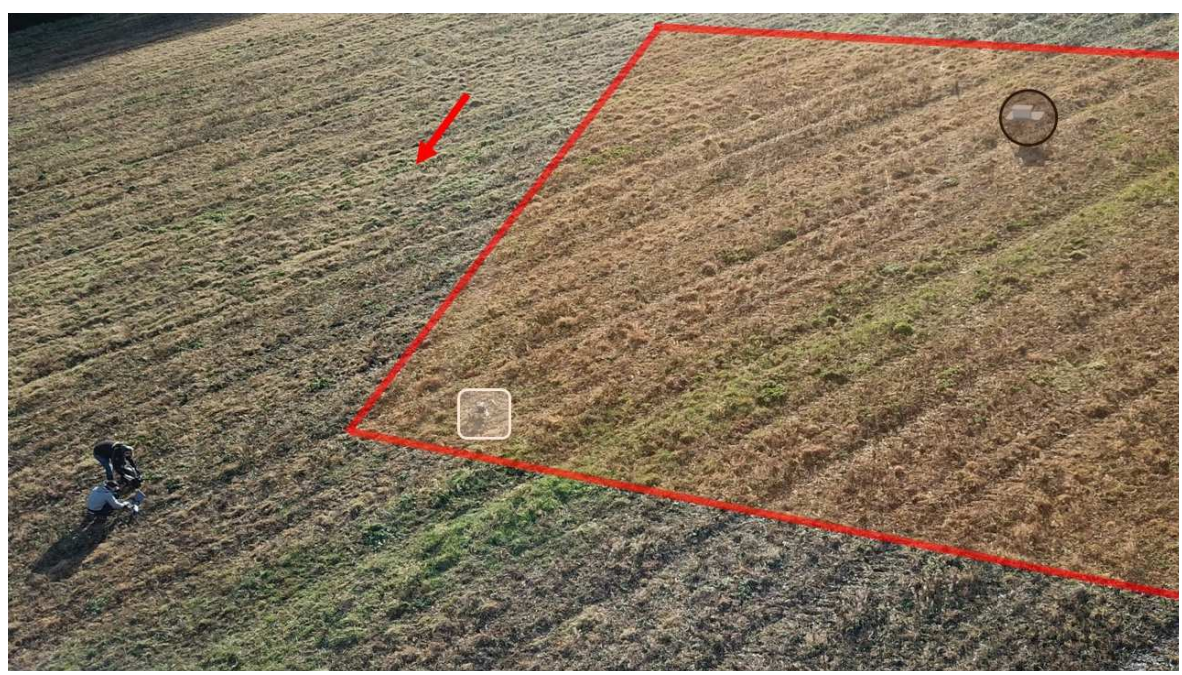

Figure 7: An example photo of the environment set-up for the experimental trials. The UAV begins the search at the white square and perform a sweep search pattern within the red area, which represents the prior distribution for the location of the diffusive source. The true position of the source is indicated by the black circle and the wind direction is implied by the red arrow.

\subsection{Implementation remarks}

In this section, the details on the implementation of the source estimation algorithm from Section 5 are outlined. This includes the specification of the prior distributions used to initialise the algorithm and the control of the UAV to collect the observational data. 


\subsubsection{Prior distributions}

Initial distributions must be set for all of the parameters in the source vector $\Theta_{k}$. Where possible the distributions are set based off sensory data, for example, the wind speed and direction distributions can be assigned from meteorological measurements. The prior distributions should reflect information known about the release, or lack thereof. For example, intelligence may exist as to the possible whereabouts of the source location, or there may exist some known bounds on the rate of emission. To assess the algorithm in realistic conditions, it is assumed, in the trials, that there is little information known about the release beforehand. The prior distributions are summarised as follows:

- The prior distributions for the location of the source $\left[p_{0}\left(x_{s}\right), p_{0}\left(y_{s}\right), p_{0}\left(z_{s}\right)\right]$ were set to uniform within the domain $\Omega$. The size of the domain is the key input of a user, essentially, it is the area in which to search for the source. Multiple domain sizes were used during the experiments, ranging from $42 \times 42 \mathrm{~m}$ to $15 \times 15 \mathrm{~m}$.

- A gamma distribution was used as the prior for the emission rate $p_{0}\left(q_{s}\right)=\mathcal{G}(1,5)$. This is a long tailed distribution to account for a large amount of uncertainty in the emission rate of the source. This prior was fixed during all of the experimental trials.

- The meteorological variables $\left[p_{0}\left(\phi_{s}\right), p_{0}\left(u_{s}\right)\right]$ were assigned Normal distributions $\mathcal{N}(\mu, \sigma)$ upon initialisation of the algorithm. In the future, the meteorological variables should be measured in-situ, on-board the UAV, in order to alleviate dependence on other data sources.

- The dispersion parameters $\left[\zeta_{s 1}, \zeta_{s 2}\right]$ were given uniform distributions with an appropriate range.

\subsubsection{UAV control}

The UAV executes a systematic sweep search pattern to collect spatial temporal measurements of the gas concentration. The search pattern can be generated using coverage path planning algorithms. As the flight path is fixed, the flight could have been pre-planned and uploaded to the autopilot using some mission planning software. However, in order to facilitate on-line, more informative planning in the future, the position demands were sent directly from the laptop during flight. The control of the UAV was made fully autonomous by utilising the dji_sdk ROS package. This included take-off, landing and the uniform sweep flight pattern. Upon take-off, the minimum altitude of the UAV was set to $1.2 \mathrm{~m}$ above ground, measured by ultrasonic sensors, to minimise the chance of a collision. Manual override was also possible throughout the experiments. 
At each time step, the UAV would hover to take an averaged measurement of the concentration. The sample duration was set to 5 seconds. This was a short amount of time compared to source term estimation methods incorporating static sensors, where it is more common to sample for a few minutes. This sampling time was chosen as a trade-off between the measurement accuracy and search time.

After the sample is collected and the source parameter estimates are updated, the UAV would proceed to the next measurement location defined by the uniform sweep pattern. The control of the UAV, to the next position, was handled autonomously by the dji_sdk ROS package. The incremental step size between each measurement location was set to $3,4,5$ or $6 \mathrm{~m}$.

\subsection{Illustrative run}

An illustrative run of an experiment, Trial 25, is given in Fig 8. Overlaid on a map of the experimental field, the figure shows: the flight path of the UAV executing the sweep search pattern at various snapshots in time; the measurements at each sampling location; the true position of the source; and an indication of the wind direction. In this example the GP model was used as the underlying dispersion model in the estimation algorithm. To begin the search, the system is initialised at discrete time step $k=0$ as shown in Fig 8 a. The starting position of the UAV is indicated by the white square, the source is given by the black circle, and the red arrow points in the direction of the wind. The large number of red dots represent the random sample approximation used in the sequential Monte Carlo algorithm at the current time step, which in this figure, approximates the prior distribution. Each dot represents a weighted source term realisation $\left\{\Theta_{k}^{(i)}, w_{k}^{(i)}\right\}$, where only the marginalised position estimates are visualised in the figure. Figures $8 \mathrm{~b}, 8 \mathrm{c}$ and $8 \mathrm{~d}$ show the trajectory of the UAV, given by the white line, and the update of the Monte Carlo samples at time steps, $k=6,16$ and 36 . The white circles indicate the positions where the UAV hovered to collect an averaged measurement from the gas detector; their size is representative of the measured value.

The illustrative run (Trial 25) was conducted in relatively high wind $(8 \mathrm{~m} / \mathrm{s})$ and neutrally stable atmospheric conditions, characterised by Pasquill's stability class D [Pasquill, 1961]. The search area was a 25x25m square in which measurements were taken at $5 \mathrm{~m}$ intervals at $1.2 \mathrm{~m}$ altitude. The sub figures in Fig 8 , show how the estimate of the source location is narrowed down significantly in response to the gas measurements. Positive detections had a larger effect on the posterior distribution as they were associated with less uncertainties than zero sensor readings as had been reflected in the respective likelihood function in Eq. (10). The location of the source was narrowed down more quickly in the crosswind direction than upwind, as seen in Fig 8c. This is an expected attribute due to the concentration distributions and characteristics of both of the underlying 


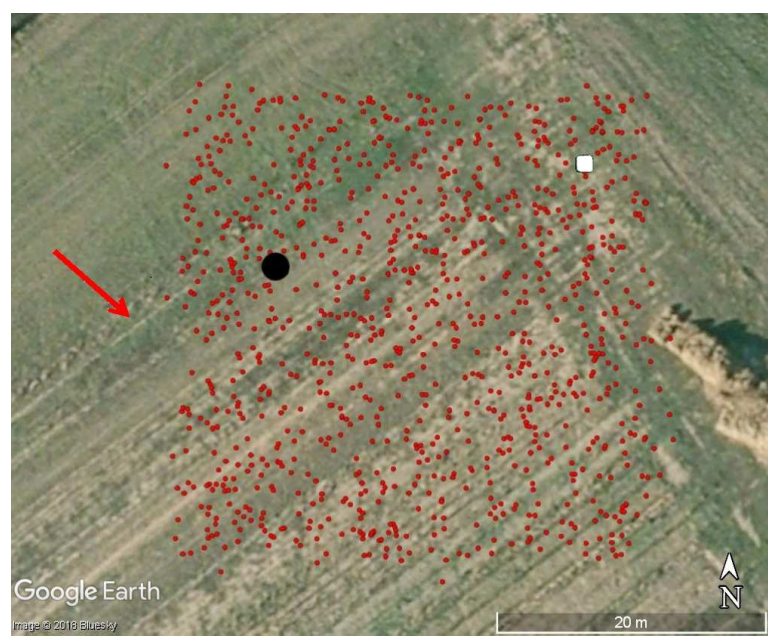

(a) Estimate at $\mathrm{k}=0$

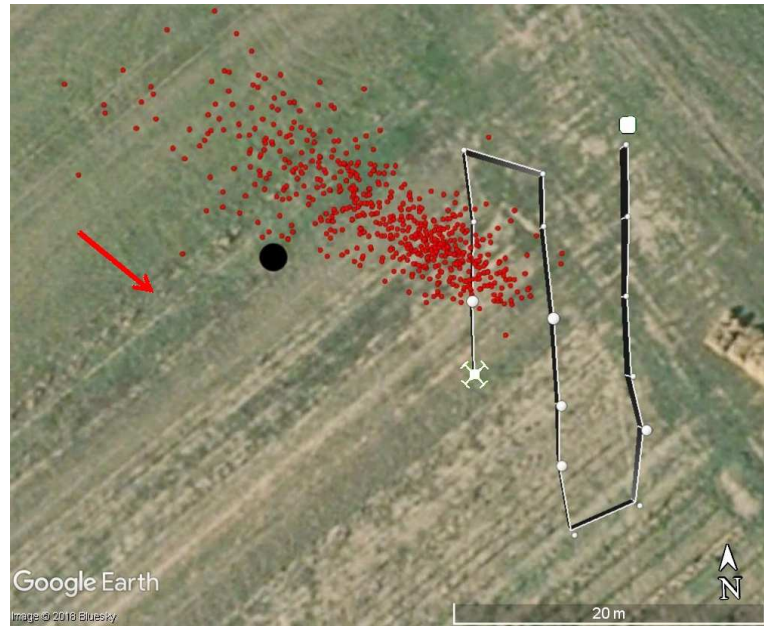

(c) Estimate at $\mathrm{k}=16$

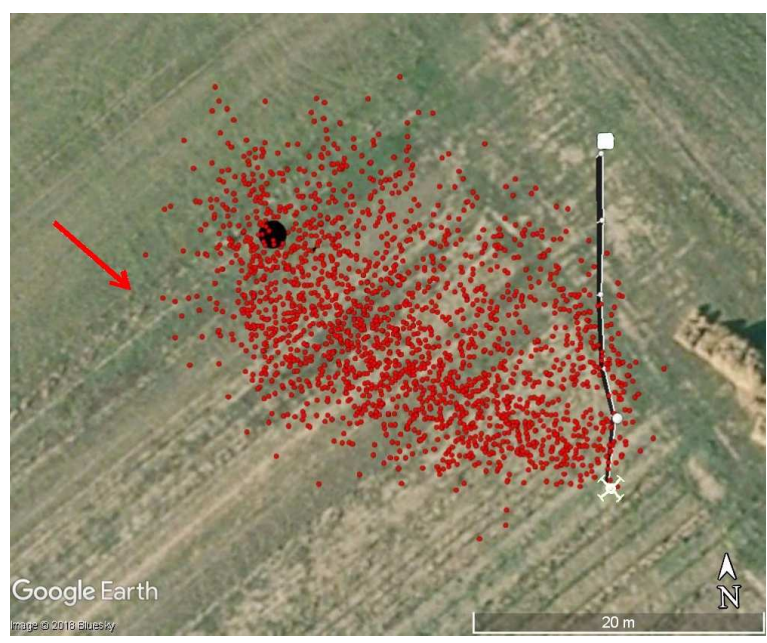

(b) Estimate at $\mathrm{k}=6$

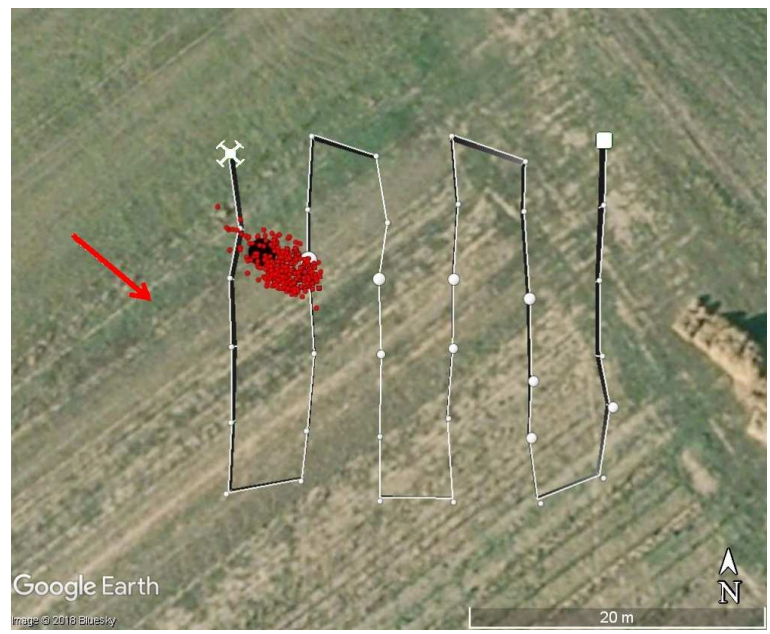

(d) Estimate at $\mathrm{k}=36$

Figure 8: Example run of the algorithm at discrete time steps: (a) k=0, (b) k=6, (c) k=16 and (d) k=36. The white line indicates the path of the UAV and the black circle represents the true position of the source. The white square and quadrotor symbol indicate the starting and current location of the UAV. The red dots represent the random sample approximation of the source parameter estimates at the current time step and the red arrow indicates the wind direction.

dispersion models, where uncertainties and correlations in the wind speed and source strength incur a lot of uncertainty in the upwind location of the source.

The result of the illustrative run is summarised in Fig 9, in a manner that is used for comparisons in the results section. Figure 9a shows the resulting flight path (white line), wind direction (red arrow) and marginalised posterior estimate of the source location (heat map). The Monte Carlo samples used in Fig 8d are replaced by a heat map to display the posterior estimate more clearly. The starting and ending positions of the UAV are given by the white square and diamond. The true position of the source is indicated by the black circle filled with a white cross and the algorithms mean estimate is given by the hollow black 


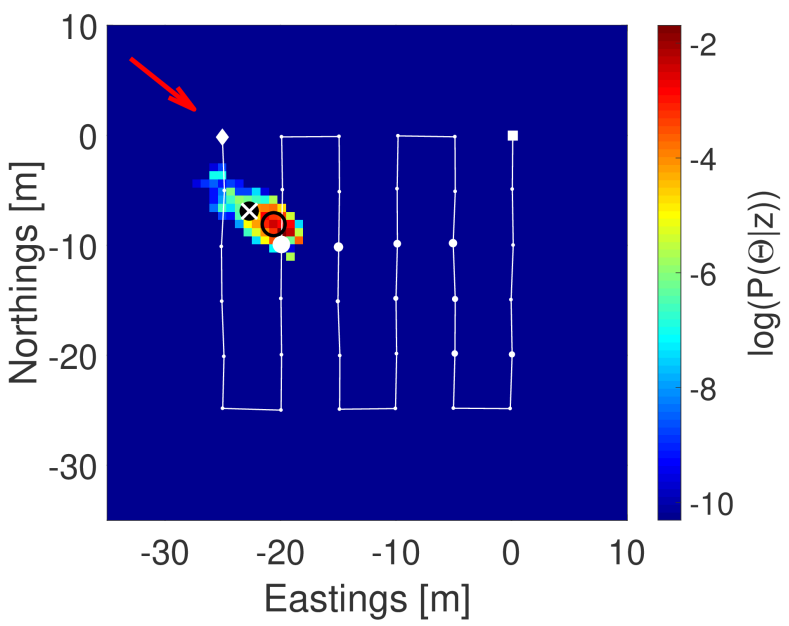

(a)
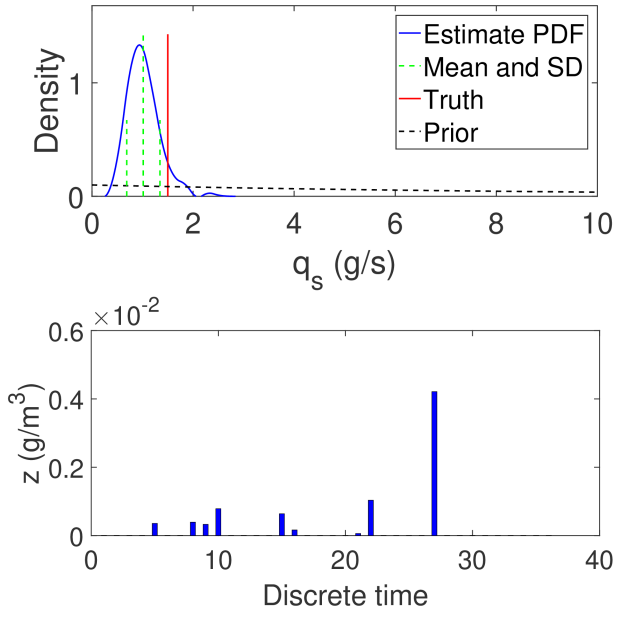

(b)

Figure 9: Summary of the illustrative run (Trial 25). (a) The resulting UAV path (white line), gas sensor measurements (white dots), wind direction (red arrow), true source position (white cross), mean source estimate (black circle) and probability density (heat map). (b) Upper: Emission rate PDF (blue curve), truth (red line), prior (dashed black curve), mean and standard deviation (dashed green lines). (b) Lower: Sensor measurements at discrete time steps.

circle. Figure $9 \mathrm{~b}$ shows the probability density estimate of the emission rate of the source, $p\left(q_{s} \mid \mathbf{z}_{1: k}\right)$, and the measurement data during the flight, $\mathbf{z}_{1: k}$. The blue curve represents the PDF of the emission estimate with mean and standard deviation indicated by the vertical dashed green lines. The black dashed curve shows the prior distribution provided to the algorithm, and the true value is given by solid red line. Bars in the lower figure of $9 \mathrm{~b}$ indicate the measurement at the discrete time step. In this example the position estimate of the source was very accurate, with only a $2.43 \mathrm{~m}$ euclidean error. The emission estimate was also accurate, but underestimated by $0.58 \mathrm{grams} / \mathrm{s}$.

\subsection{Results}

In total, 27 experimental trials were conducted to test the system in the fairly realistic setting described. The experiments were conducted at various flight altitudes, wind speeds and scales. The results, using both the IP and GP dispersion models, are summarised in Table 1. The table includes details on the scale of the experiments, the step size or incremental distance between sensor measurements, the UAVs flight altitude, the duration of an experiment and the wind speed. The output euclidean position error and the absolute emission errors are shown, where the true values are compared with the means of the estimation algorithm.

Overall, the euclidean error was small considering the scale of the experiments, the severe amount of uncertainty in the dispersion process, and some uncertainty in the localisation of the UAV itself. In the majority of 
Table 1: Summary of results for the 27 experimental trials, using the GP and IP models, including the errors in the source location and emission rate estimates, and flight pattern data.

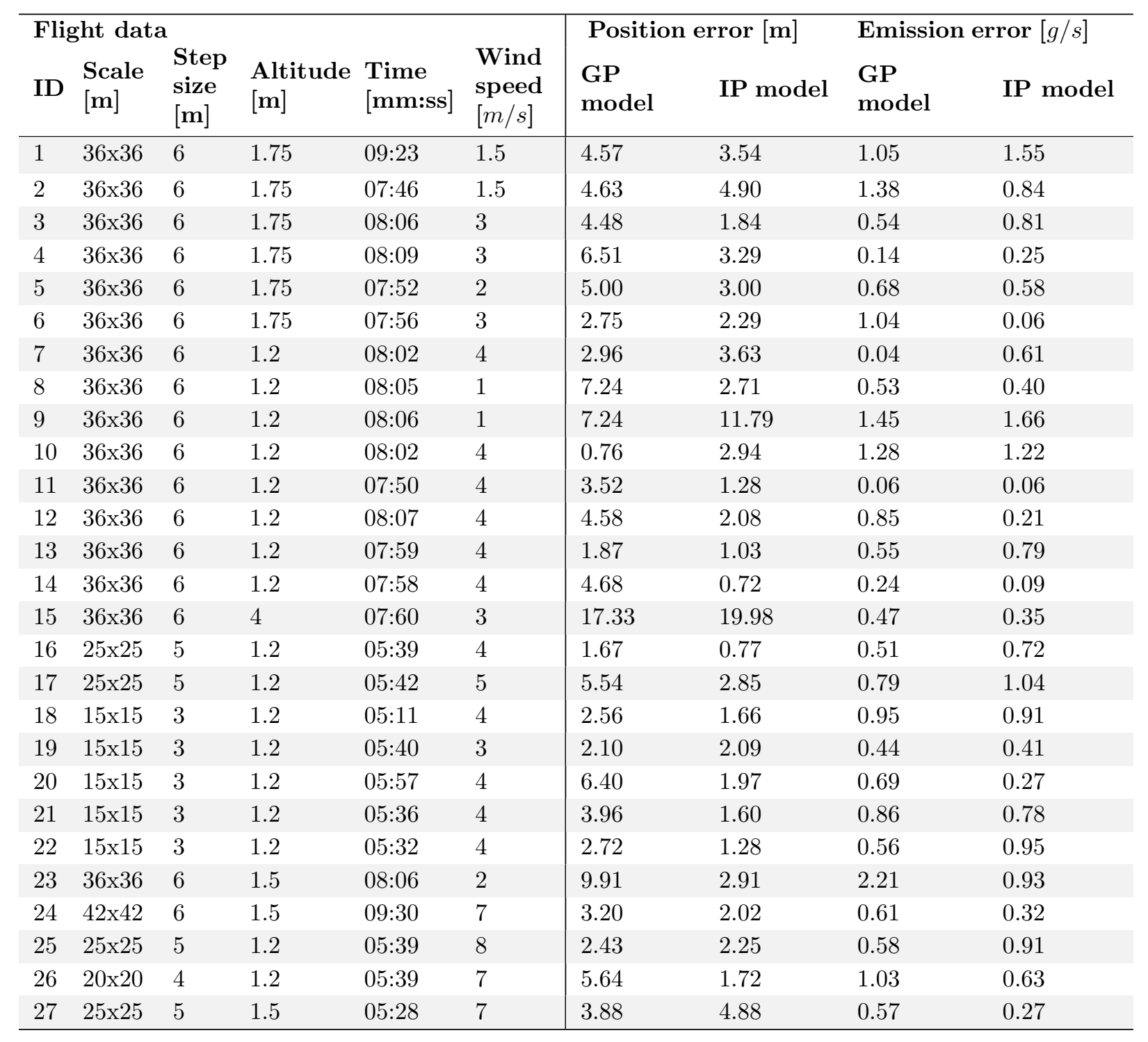

the experiments the error was noticeably within the step size used in the flight pattern. This was not always the case, due to changing meteorology and the large amount of intermittency in the gas detections in the vicinity of the source. The emission estimates from the algorithm using both models were encouraging, with errors typically under $1 \mathrm{~g} / \mathrm{s}$. Given the review on gas sensing using UAVs in Section 2, the release estimate was expected to be under predicted due to decreased readings from the sensor caused by the rotor effect. However, the outcomes of the trials were varied; featuring very accurate estimates in addition to under or over predictions. Despite the reduced measurements, which may be responsible for the under predications of the emission rates; the over predictions are expected to be a result of the shorter sampling times that were 
adopted for data collection by the UAV. This lead to significantly more volatile measurements where there is the potential to average over a period of intermittency or during a large spike in concentration; which, given a larger sampling time, would typically be accompanied by smaller readings to smooth the average. This averaging over a spike is expected to be the cause of the over prediction of the emission rate that occurred in some of the trials. Other factors that had the most effect on the estimation accuracy were the flight altitude of the UAV, the step size between taking measurements and the wind speed.

Flying at different altitudes affected the estimation performance of the system as it changed the concentration observations made by the sensor onboard the UAV. Acetone is a dense material, so at high altitudes the UAV would be outside of the plume, where it would detect nothing with the gas sensor. Example results of flights conducted at $1.2 \mathrm{~m}, 1.75 \mathrm{~m}$ and $4 \mathrm{~m}$ altitudes are shown in Fig 10, where the IP model was used in the estimation. For reference, the height of the source during the experiments was $1.4 \mathrm{~m}$. It was found, due to the density of the acetone, that at lower flight altitudes the sensor on-board the UAV picked up more positive detections, with less intermittency, which resulted in more accurate estimates of the source term with less spread, as observed in Fig 10. Note: in figure 10f, the scale of the sensor data axis is smaller for the flight conducted at $4 \mathrm{~m}$ altitude. At altitudes greater than $4 \mathrm{~m}$ there would generally be zero detections made by the gas sensor. All flights were of the same scale and step size and conducted in similar wind conditions.

The effect of the step size between measurements made by the gas sensor was as expected. The closer, more dense measurements resulted in more accurate estimates with less spread. This is illustrated in Fig 11 which shows example results of flights with samples taken at $3 \mathrm{~m}, 5 \mathrm{~m}$, and $6 \mathrm{~m}$ increments along the sweep path. All flights were conducted at $1.2 \mathrm{~m}$ altitude in similar wind conditions and the IP model was used in the estimation.

The affects the wind speed has on the estimates made by the system are twofold: 1) In stronger winds more acetone remained airborne, rather than falling to the ground, resulting in more positive detections from the gas sensor and better matching between the observations and the dispersion models which did not account for the buoyancy of the material; 2) Stronger winds are linked with greater atmospheric stability [Pasquill, 1961] which leads to more consistent meteorological conditions. Examples of experiments conducted in 1, 4 and $7 \mathrm{~m} / \mathrm{s}$ winds at similar scales and altitudes are shown in Fig 12, where the IP model was used in the estimation. Studying the figures, it is clear how the sensing characteristics of the system are much better in higher winds. Figures $12 \mathrm{a}$ and $12 \mathrm{~b}$ show the results in $1 \mathrm{~m} / \mathrm{s}$ mean wind speed on a hot sunny day which is associated with the most unstable atmospheric stability class (Pasquill's stability Class A). During this trial, the wind speed was negligible at times and the direction completely reversed. The poor sensing conditions, 


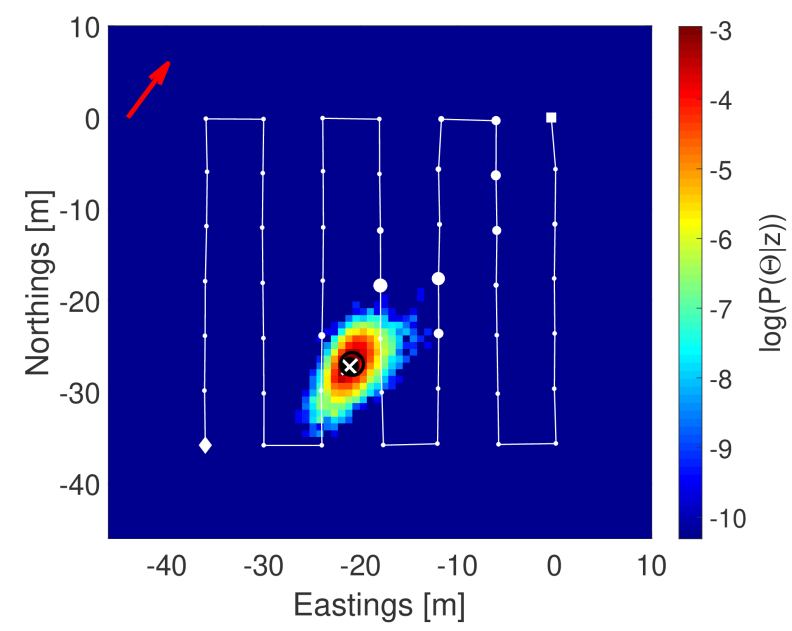

(a) Trial 14, position estimate.

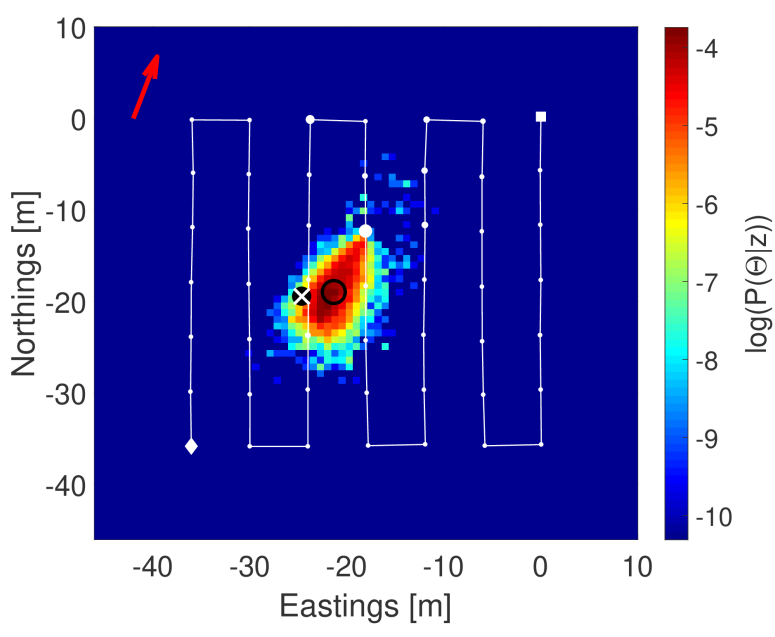

(c) Trial 4, position estimate.

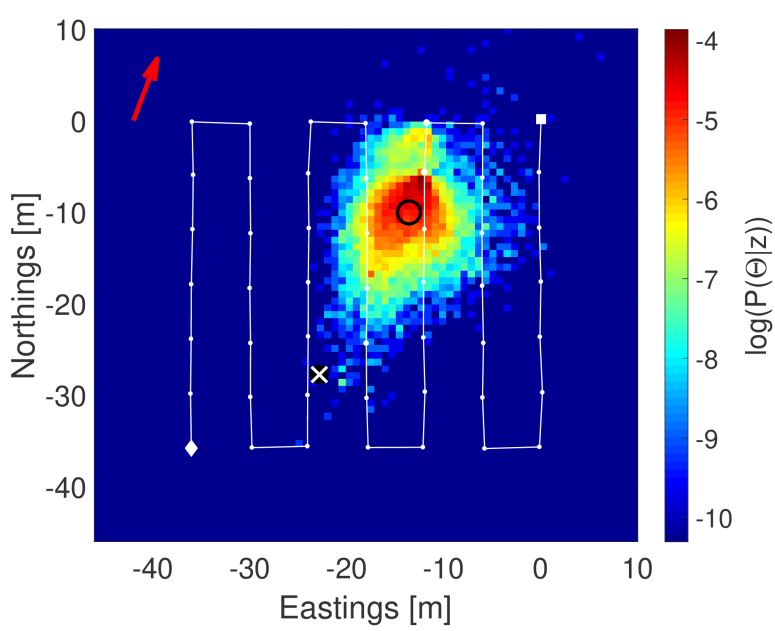

(e) Trial 15, position estimate.
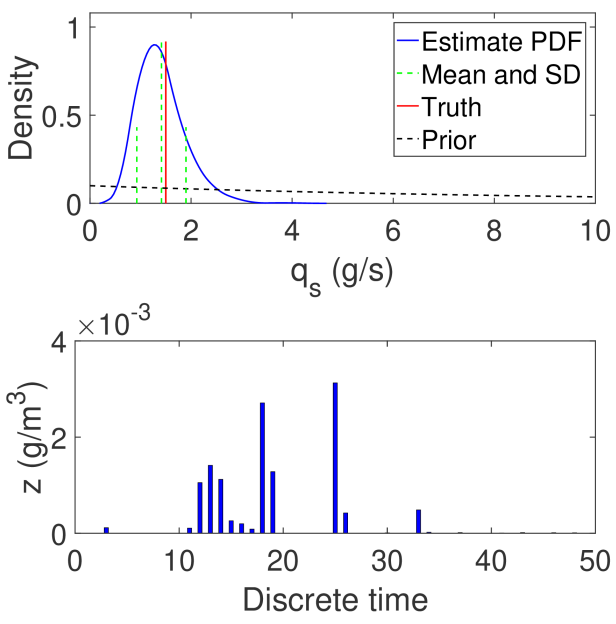

(b) Trial $14, q_{s}$ estimate and sensor data.
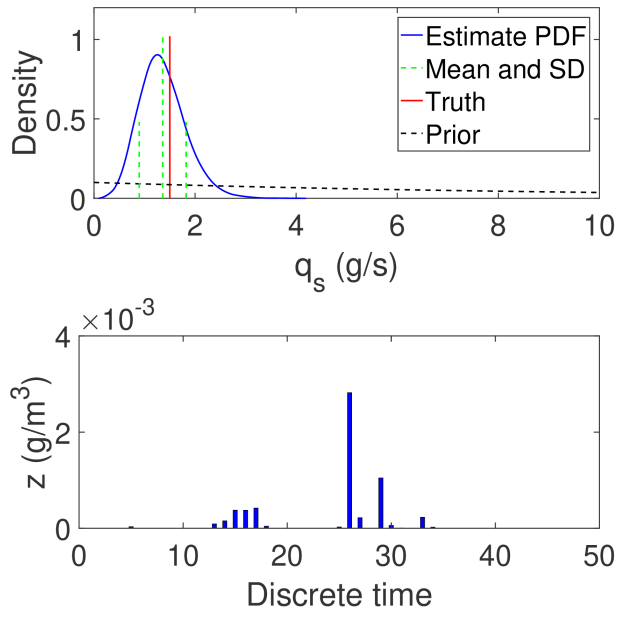

(d) Trial $4, q_{s}$ estimate and sensor data.
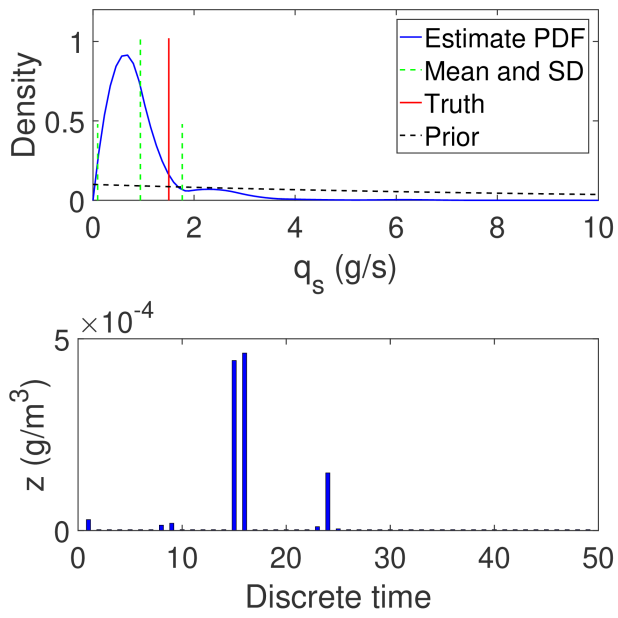

(f) Trial $15, q_{s}$ estimate and sensor data.

Figure 10: Results at altitudes (a-b) 1.2m: Trial 14, (c-d) 1.75m: Trial 4 and (e-f) 4m: Trial 15, using the IP model. The search area was $36 \times 36 \mathrm{~m}$, the step size was $6 \mathrm{~m}$ and wind speed $3-4 \mathrm{~m} / \mathrm{s}$. 


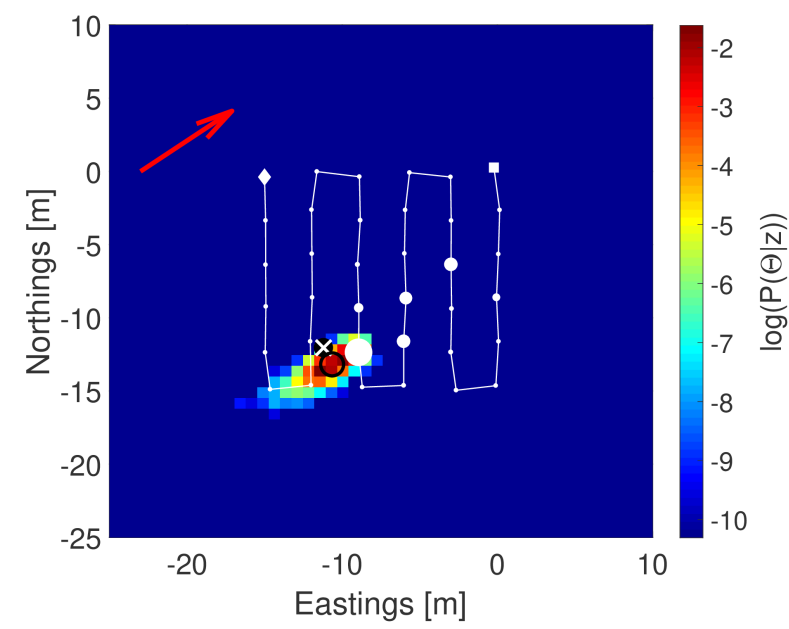

(a) Trial 22, position estimate.

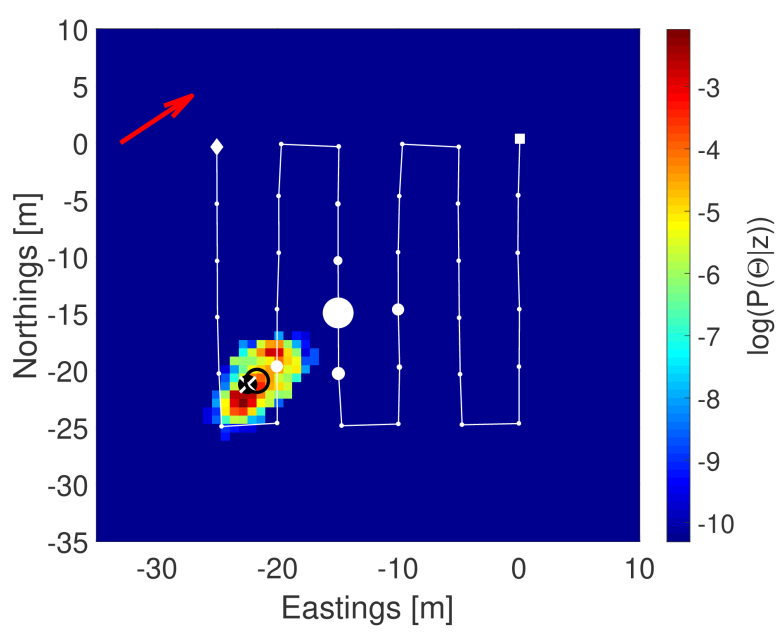

(c) Trial 16, position estimate.

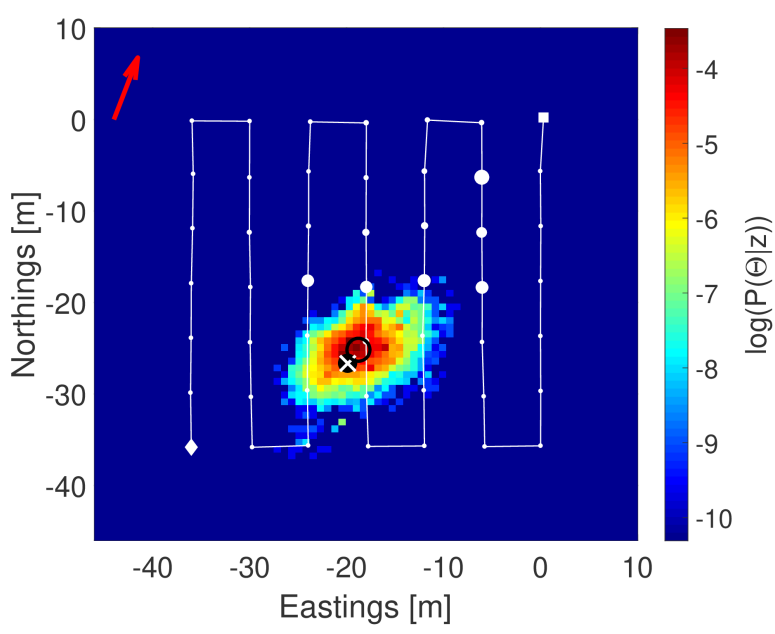

(e) Trial 12, position estimate.
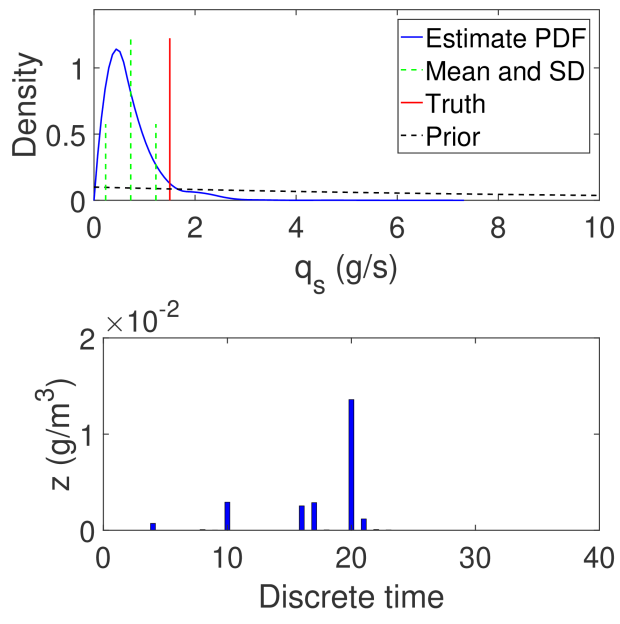

(b) Trial 22, $q_{s}$ estimate and sensor data.
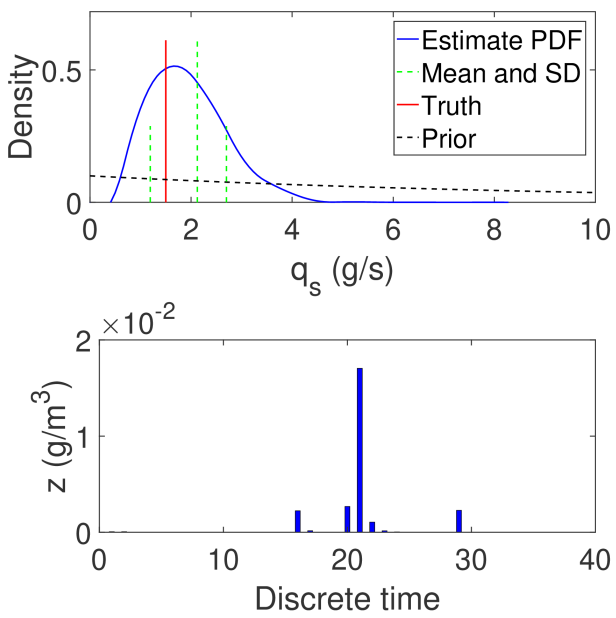

(d) Trial 16, $q_{s}$ estimate and sensor data.
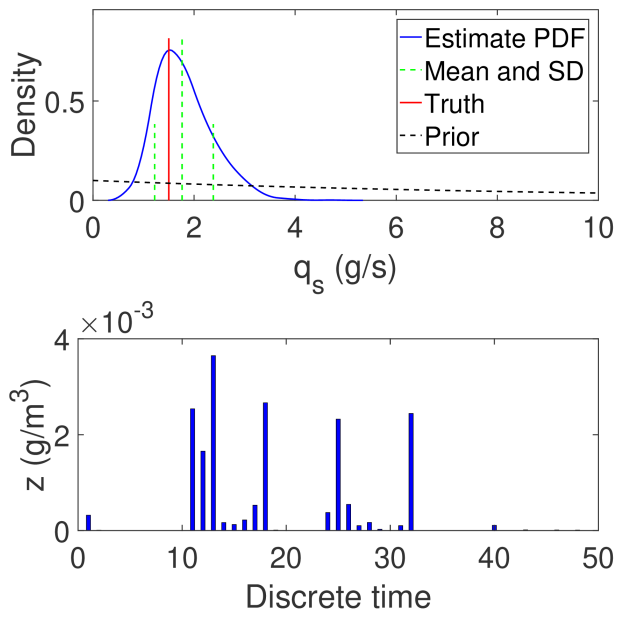

(f) Trial $12, q_{s}$ estimate and sensor data.

Figure 11: Results at step sizes (a-b) 3m: Trial 22, (c-d) 5m: Trial 16 and (e-f) 6m: Trial 12, using the IP model. The UAV altitude was $1.2 \mathrm{~m}$, the step size was $6 \mathrm{~m}$ and wind speed $4 \mathrm{~m} / \mathrm{s}$. 


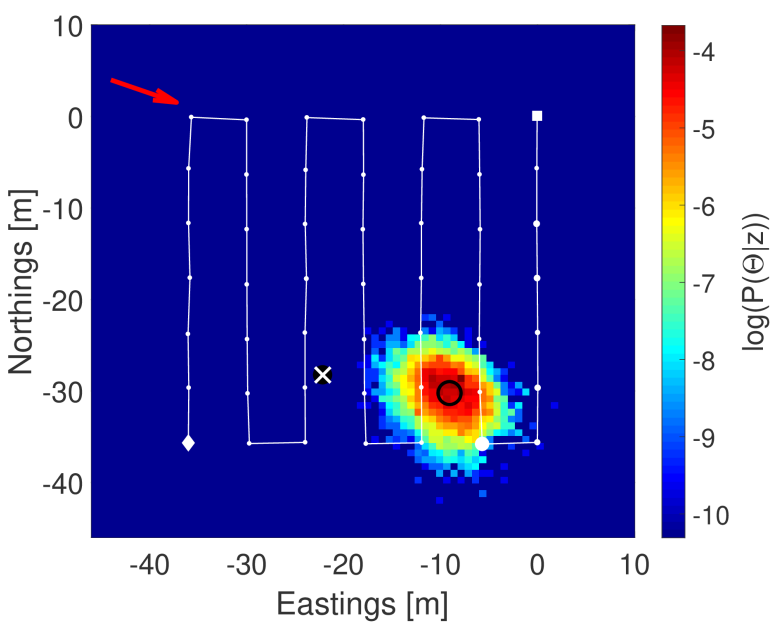

(a) Trial 9, position estimate.

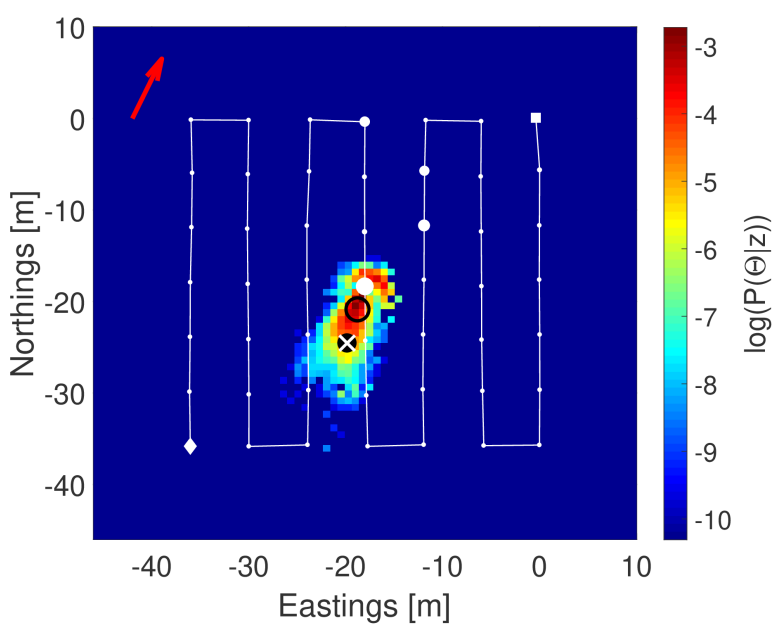

(c) Trial 7, position estimate.

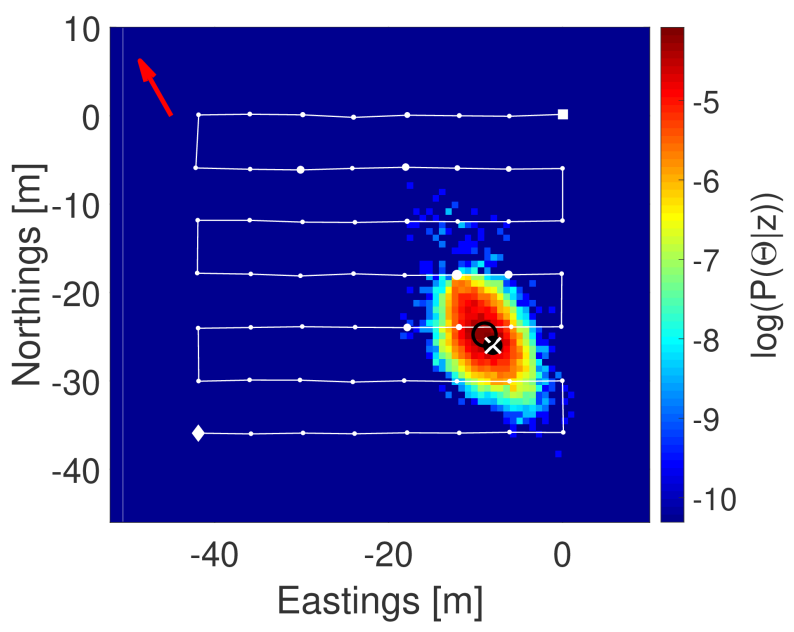

(e) Trial 24, position estimate.
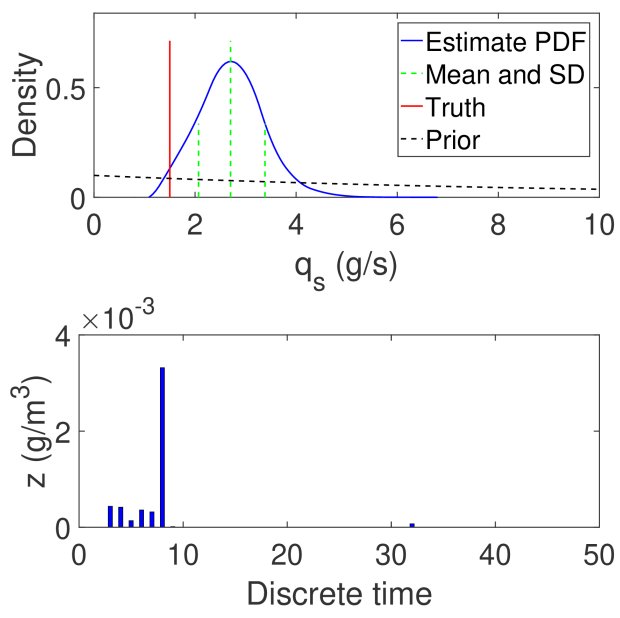

(b) Trial 9, $q_{s}$ estimate and sensor data.
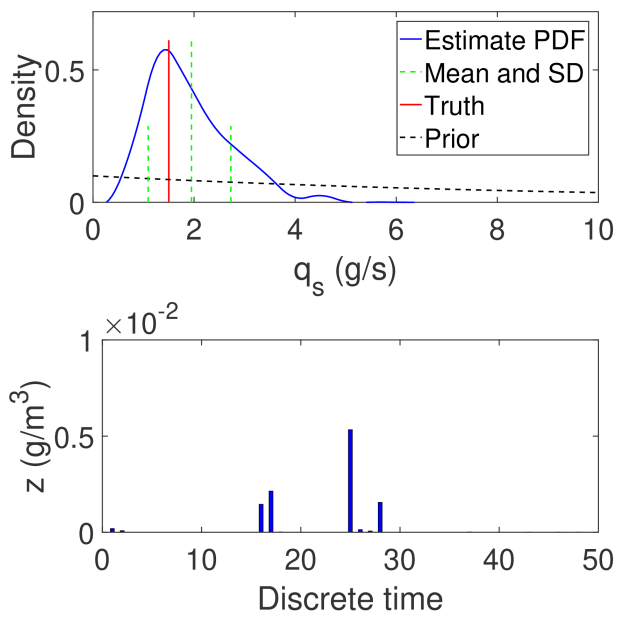

(d) Trial 7, $q_{s}$ estimate and sensor data.
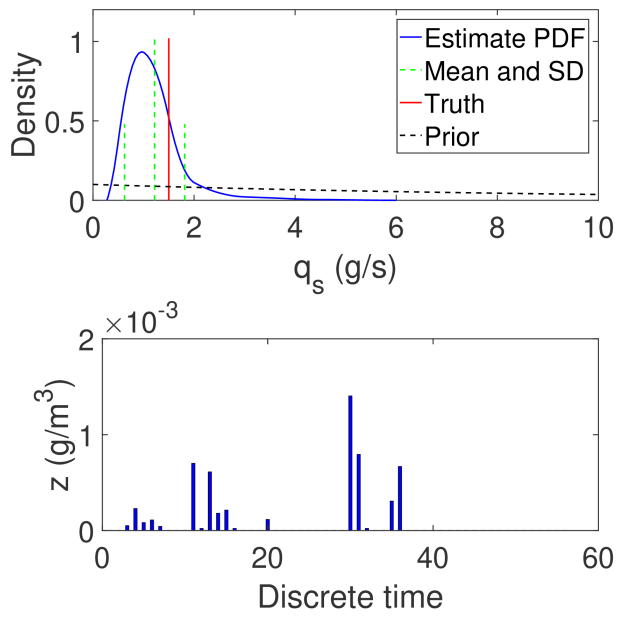

(f) Trial 24, $q_{s}$ estimate and sensor data.

Figure 12: Results at wind speeds (a-b) $1 \mathrm{~m} / \mathrm{s}$ : Trial 9, (c-d) 4m/s: Trial 7 and (e-f) $7 \mathrm{~m} / \mathrm{s}$ : Trial 24, using the IP model. The UAV altitude was $1.2-1.5 \mathrm{~m}$ and the step size was $6 \mathrm{~m}$. 
where acetone was detected only near the beginning of the flight, resulted in the inaccurate estimate of the source location. In higher wind, as shown in figures $12 \mathrm{e}$ and $12 \mathrm{f}$, the wind direction and speed was more consistent producing a better defined plume. The corresponding source position and emission estimates were very accurate with errors of $2.02 \mathrm{~m}$ and 0.32 grams $/ \mathrm{s}$.

The root mean squared errors (RMSE) and standard deviations (SD) of the position and emission estimates for the GP and IP models are shown in Table 2. The RMSEs and SDs are split among all the trials and subsets of the trials corresponding to different flight altitudes, step sizes and wind speeds. The IP model outperformed the GP model in all conditions with regards to the position estimates and in the majority of conditions for the emission estimates. The difference in performance is expected to be caused by the characteristics of the models near the source, for which the IP model more closely matched the data collected by the UAV in the particular experiments conducted in this paper. The limiting characteristic of the GP model was its approximation of the width of the plume near the source, which was often wider than anticipated by the model. The wind speed appeared to have the greatest effect on the RMSE and SD of the estimates. In higher wind speeds the system produced consistently accurate estimates, resulting in low values of RMSE and SD. Small wind speeds $(\leq 3 \mathrm{~m} / \mathrm{s})$, which correspond to significantly more unstable atmospheric conditions, resulted in the most inaccurate and variable estimates. Considering Table 2, the best results are obtained with a smaller step size. However, a larger step size did not impede the results to the extent of weak wind speeds or flying at higher altitudes that are on the edge of the plume.

A common method to assess the performance of source localisation systems is the rate of successful localisations [Marques, 2011]. This metric introduces some ambiguity with regards to the definition of a successful localisation, which is usually given as a certain distance between the estimated and true source positions. Therefore, to provide a more explicit idea of the performance of the system, the success rate is plotted for various values of success criteria in Fig 13, where the success criteria is given as a range of euclidean errors in the source position estimate. The success rate is shown for estimations made using the IP and GP models. Note: The result of Trial 15 (conducted at $4 \mathrm{~m}$ altitude) was neglected as this was used to demonstrate the adverse effect of high altitudes. The figure shows the results of the remaining 26 trials and the subset where the wind speed was greater than $3 \mathrm{~m} / \mathrm{s}$.

To conclude the results, the parameters of a gaseous release into the atmosphere have been estimated using point measurements of concentration from an autonomous UAV equipped with a gas detector. The results in Table 1 show accurate estimates for the source location and its emission rate obtained using the Bayesian inference method described in Section 5. Both dispersion models performed well but the IP model was more 
Table 2: RMSE and SD in the position and emission rate estimates using the GP and IP dispersion models.

\begin{tabular}{l|lclc}
\hline & \multicolumn{2}{|l}{ Position RMSE (SD) $[\mathbf{m}]$} & \multicolumn{2}{l}{ Emission RMSE (SD) $[\mathrm{g} / \mathrm{s}]$} \\
Data subset & $\begin{array}{l}\text { GP } \\
\text { model }\end{array}$ & IP model & $\begin{array}{l}\text { GP } \\
\text { model }\end{array}$ & IP model \\
\hline All data & $4.75(3.24)$ & $3.35(3.93)$ & $0.75(0.47)$ & $0.65(0.43)$ \\
Step size $=6 \mathrm{~m}$ & $5.37(3.79)$ & $4.08(4.78)$ & $0.77(0.58)$ & $0.63(0.50)$ \\
Step size $=5 \mathrm{~m}$ & $3.38(1.71)$ & $2.69(1.70)$ & $0.61(0.12)$ & $0.73(0.34)$ \\
Step size $=3 \mathrm{~m}$ & $3.55(1.74)$ & $1.72(0.32)$ & $0.70(0.21)$ & $0.66(0.31)$ \\
Height $\geqslant 1.5 \mathrm{~m}$ & $5.22(2.17)$ & $3.33(1.10)$ & $0.95(0.64)$ & $0.66(0.48)$ \\
Height $<1.5 \mathrm{~m}$ & $3.88(2.00)$ & $2.45(2.51)$ & $0.67(0.39)$ & $0.69(0.42)$ \\
Wind speed $\leqslant 3 \mathrm{~m} / \mathrm{s}$ & $6.45(4.43)$ & $4.65(5.45)$ & $0.85(0.60)$ & $0.62(0.43)$ \\
Wind speed $>3 \mathrm{~m} / \mathrm{s}$ & $3.86(1.76)$ & $2.63(2.58)$ & $0.69(0.38)$ & $0.66(0.43)$ \\
\hline
\end{tabular}

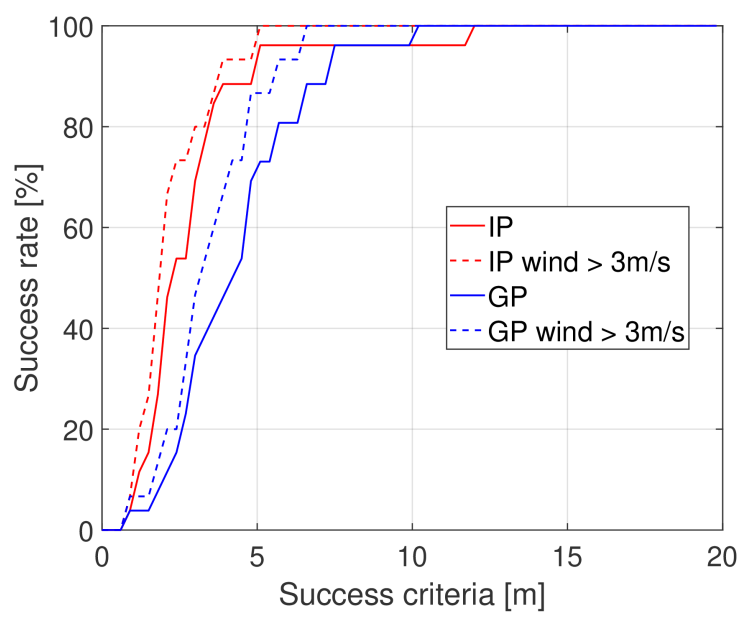

Figure 13: Success rates of the system.

accurate in the experimental conditions described in this paper. The overall accuracy of the source estimates was dependant on the measurements taken from the UAV, and how they matched the chosen ATD model featured in the likelihood function. The measurement data was affected by the meteorology, the altitude of the UAV, and the size of the increments between sampling the gas concentration.

\section{Discussions and lessons learned}

The results of the experimental trials presented are, to the best of the authors knowledge, the first time an autonomous UAV has been used to collect gas concentration measurements to estimate the source term (location and strength) of a release. Given such an immature area of work, there are valuable observations and lessons learned during the experiments that will be described in this section. These are related to the 
position of the UAV, gas sensing with an aerial vehicle, and the local meteorology.

\subsection{UAV altitude}

Given the chosen method to estimate the parameters of the release, it is important to know the location of the gas concentration measurements accurately, so that they can be related to the predictions from a model. In the horizontal directions, this was achieved through fusion of GPS data, IMU data and the guidance system on-board the aircraft. The altitude of the UAV was more challenging. When relying on barometric data, the UAV altitude could drift by a couple of meters. Although this is not very large, on the small scale of the experiments it would have an adverse effect on the results, especially given the low altitude of the UAV and the small size of the acetone plume. Down facing ultrasonic sensors of the guidance system provided an accurate estimate of the height above ground, however, this source of information was only suitable for level terrain. Consequently, this assumption was made during the experiments.

\subsection{Gas sensing with a UAV}

The rotors of the UAV did reduce the concentration readings of the gas detector. This was visible when the UAV was stationary on the ground and the rotors would turn off and on. Despite this, the results of the experiments were extremely positive, with the source emission rate only slightly underestimated. As discussed in Section 2.1, the sensor inlet could be moved outside the region of influence of the rotors or, in future research, a new model for the sensor response could be formulated. In addition, during visualisation experiments performed using coloured smoke, it was found that the UAV seemed to split the plume; as captured in Fig 14. This is a feature that may become important in path planning research in the future or when cooperating multiple vehicles.

\subsection{Gas Buoyancy}

The diffused acetone is dense, reducing how the gas would rise from the source. This results in low altitudes required by the UAV in order to make contact with the plume, and it causes the gas dispersion to be less buoyant than modelled by the simple transport equations considered in this paper. Adding the effect of buoyancy to the model could improve the accuracy of the estimation. This could be included as a parameter with uncertainty, and inferred as part of an extension of the source vector $\Theta_{k}$. 


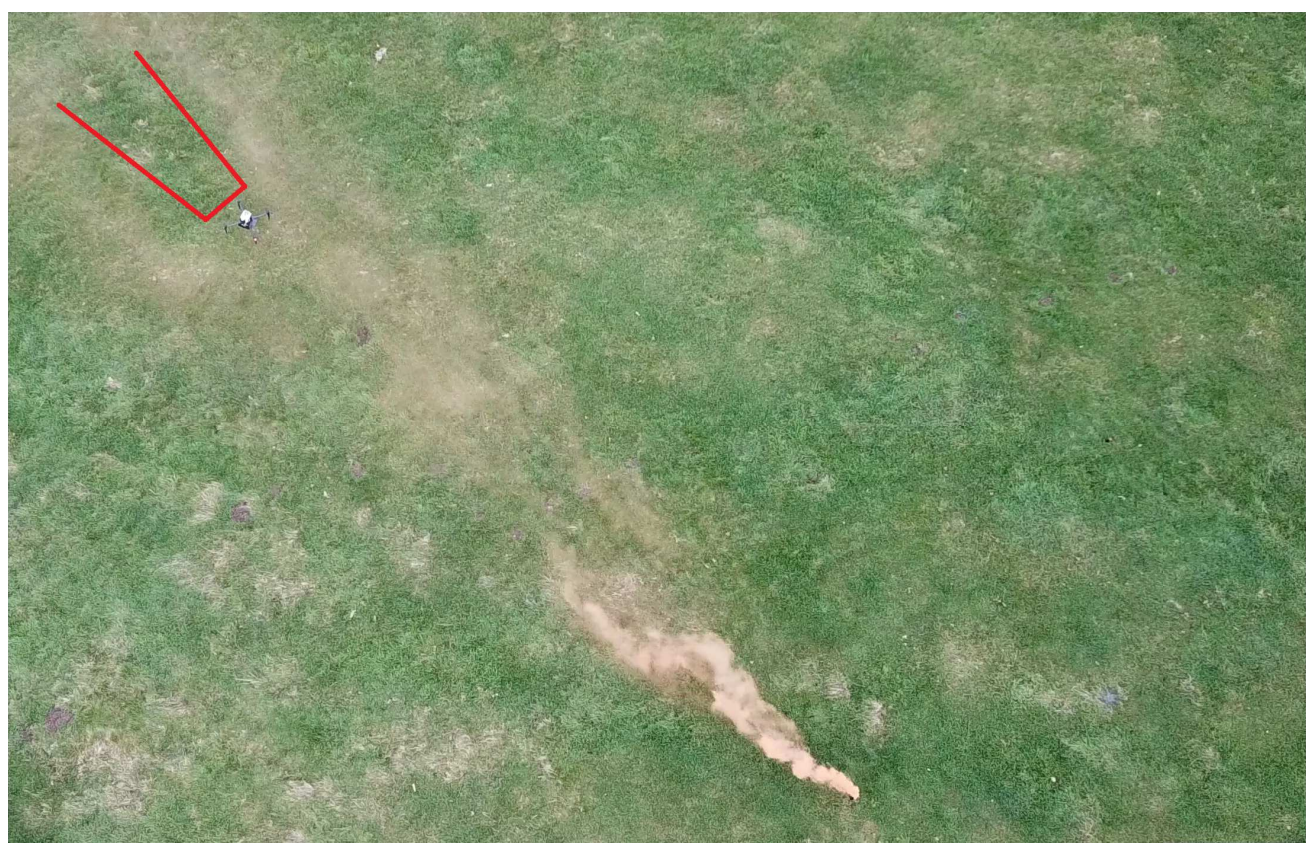

Figure 14: Visualisation of the UAVs effect on the gas dispersion using coloured smoke. A gap in the plume can be seen directly downwind of the platform, highlighted by the red lines.

\subsection{Changing meteorology}

In this work, and commonly in the literature, source term estimation algorithms for atmospheric releases assumed steady state conditions in the underlying dispersion model. This assumption holds in simulations and in wind tunnel datasets. Outside of simulation the condition is often not fulfilled, particularly on highly unstable, low wind speed conditions, where the direction of the wind is much more random. This incurs a large error on the estimation of the source location. This may be overcome by employing a different model, such as a Gaussian Puff, which does not make a steady state assumption. However, some assumptions on the wind field would still be required, and the model would be more computationally expensive.

\subsection{Sampling time and step size}

The sampling time, or how long the UAV hovers to take an averaged measurement of concentration, had been set to a fixed value based on a trade-off between search time and performance. The step size, or movement distance between each sample, was selected in a similar manner. Given a larger area to search, it may be necessary to reduce the sampling time or increase the movement distance so that the UAV can search the area in less time. In future work, these parameters of the planning algorithm should be selected adaptively based on the current information available, or even removed altogether by considering observations from a 
continuously moving platform.

\section{Conclusions}

A system has been described to enable estimation of the parameters of a dispersive atmospheric release using a UAV. Given an area to search, which could potentially be drawn over a map on a tablet, the system is able to estimate the parameters of the source term within the area including the origin of the release and its emission rate. Bayes' theorem was used in order to account for the large amount of uncertainty concerning the concentration observations from the gas detector, the meteorological parameters, and the representativeness of the underlying dispersion models. The set-up of a UAV has been described to enable the experimental validation of the algorithm in an outdoor open field. To the best of the authors knowledge, this has been the first experimental testing of a source term estimation algorithm using an autonomous UAV, where a static network of gas detectors has been used in the past. Extensive experiments were conducted in various meteorological conditions and with different sweeping flight path configurations. Using the data generated from a total of 27 experiments, the effect of the UAVs attitude, the incremental step size between taking measurements, and the wind speed was assessed. It was found, in the experiments conducted, which used a relatively dense material as a source, that the system performed better, in terms of estimation accuracy, flying at lower altitudes and in higher wind speeds (stronger atmospheric stability [Pasquill, 1961]). The wind speed, and hence the atmospheric stability, was found to have a significant effect on the accuracy of the algorithm and the SD of the estimation errors. As expected, the smaller incremental step sizes between gas measurements, resulted in more accurate source estimates with less spread. However, this would incur significant penalties on the size of the area covered over time. Additionally, two simple, fast running dispersion models were compared using the data from the unique experiments conducted in the paper. Overall, the Isotropic plume model noticeably outperformed the standard Gaussian model. This is expected to be due to the characteristics of the models in the vicinity of the source, suggesting the IP model is more appropriate for estimation on a smaller scale. Overall, the experimental results demonstrated strong performance of the system making it closer to use in real scenarios.

There are several areas for future research that could improve the performance of the system or expand its capabilities. Besides the development and testing of a more informative flight pattern that capitalised on the information gained during flight; the search time and potentially the accuracy of the source term estimate could be improved by removing the requirement to stop, or hover, to take an averaged sample of the hazard. This may be achieved by taking a sensor average whilst moving, or by using the instantaneous observations of 
the sensor. This will introduce new problems due to the dynamics of the sensor, and designing an appropriate likelihood function to account for the new sampling technique. The source term estimation methods in the past, which used static sensors, had much longer sampling times which resulted in less intermittency and spread in the measurements. Finally, the experiments in this paper were restricted to an open area without obstacles or clutter, and a continuously emitting source. The system should be extended to handle more challenging environments such as urban or industrial areas, a source or sources of finite duration, and changes in atmospheric conditions.

\section{Acknowledgements}

This project was funded by the UK Ministry of Defence via the Defence and Security Accelerator under project number ACC500113. The authors would like to express their gratitude to colleagues Matthew Coombes and Pawel Ladosz of Loughborough University for their assistance during the experimental trials.

\section{References}

Adler, J. (1966). Chemotaxis in bacteria. Science, 153(3737):708-716.

Allwine, K., Leach, M., Stockham, L., Shinn, J., Hosker, R., Bowers, J., and Pace, J. (2004). J7. 1 overview of joint urban 2003-an atmospheric dispersion study in oklahoma city.

Alvarado, M., Gonzalez, F., Erskine, P., Cliff, D., and Heuff, D. (2017). A methodology to monitor airborne pm10 dust particles using a small unmanned aerial vehicle. Sensors, 17(2):343.

Alvarado, M., Gonzalez, F., Fletcher, A., and Doshi, A. (2015). Towards the development of a low cost airborne sensing system to monitor dust particles after blasting at open-pit mine sites. Sensors, 15(8):19667-19687.

Aurell, J., Mitchell, W., Chirayath, V., Jonsson, J., Tabor, D., and Gullett, B. (2017). Field determination of multipollutant, open area combustion source emission factors with a hexacopter unmanned aerial vehicle. Atmospheric Environment, 166:433-440.

Bennetts, V. H., Schaffernicht, E., Stoyanov, T., Lilienthal, A. J., and Trincavelli, M. (2014). Robot assisted gas tomographylocalizing methane leaks in outdoor environments. In Robotics and Automation (ICRA), 2014 IEEE International Conference on, pages 6362-6367. IEEE. 
Champagne, L., Carl, R. G., and Hill, R. (2003). Agent models ii: search theory, agent-based simulation, and u-boats in the bay of biscay. In Proceedings of the 35th conference on Winter simulation: driving innovation, pages 991-998. Winter Simulation Conference.

Chang, C.-C., Wang, J.-L., Chang, C.-Y., Liang, M.-C., and Lin, M.-R. (2016). Development of a multicoptercarried whole air sampling apparatus and its applications in environmental studies. Chemosphere, 144:484-492.

Chino, M., Nakayama, H., Nagai, H., Terada, H., Katata, G., and Yamazawa, H. (2011). Preliminary estimation of release amounts of $131 \mathrm{i}$ and $137 \mathrm{cs}$ accidentally discharged from the fukushima daiichi nuclear power plant into the atmosphere. Journal of nuclear science and technology, 48(7):1129-1134.

Derezinski, D. D., Lacy, M. G., and Stretesky, P. B. (2003). Chemical accidents in the united states, 1990-1996. Social science quarterly, 84(1):122-143.

Efthimiou, G. C., Kovalets, I. V., Venetsanos, A., Andronopoulos, S., Argyropoulos, C. D., and Kakosimos, K. (2017). An optimized inverse modelling method for determining the location and strength of a point source releasing airborne material in urban environment. Atmospheric Environment.

Greatwood, C., Richardson, T. S., Freer, J., Thomas, R. M., MacKenzie, A. R., Brownlow, R., Lowry, D., Fisher, R. E., and Nisbet, E. G. (2017). Atmospheric sampling on ascension island using multirotor uavs. Sensors, 17(6):1189.

He, G., Zhang, L., Lu, Y., and Mol, A. P. (2011). Managing major chemical accidents in china: Towards effective risk information. Journal of hazardous materials, 187(1):171-181.

Hernandez Bennetts, V., Lilienthal, A. J., Neumann, P., and Trincavelli, M. (2012). Mobile robots for localizing gas emission sources on landfill sites: is bio-inspiration the way to go? Frontiers in neuroengineering, $4: 20$.

Hirst, B., Jonathan, P., del Cueto, F. G., Randell, D., and Kosut, O. (2013). Locating and quantifying gas emission sources using remotely obtained concentration data. Atmospheric environment, 74:141-158.

Hutchinson, M., Liu, C., and Chen, W.-H. (2018a). Information-based search for an atmospheric release using a mobile robot: Algorithm and experiments. IEEE Transactions on Control Systems Technology, $(99): 1-15$.

Hutchinson, M., Oh, H., and Chen, W.-H. (2017a). Adaptive bayesian sensor motion planning for hazardous source term reconstruction. IFAC-PapersOnLine, 50(1):2812-2817. 
Hutchinson, M., Oh, H., and Chen, W.-H. (2017b). A review of source term estimation methods for atmospheric dispersion events using static or mobile sensors. Information Fusion, 36:130-148.

Hutchinson, M., Oh, H., and Chen, W.-H. (2018b). Entrotaxis as a strategy for autonomous search and source reconstruction in turbulent conditions. Information Fusion, 42:179-189.

Ishida, H., Wada, Y., and Matsukura, H. (2012). Chemical sensing in robotic applications: A review. IEEE Sensors Journal, 12(11):3163-3173.

Jaynes, E. T. (2003). Probability theory: The logic of science. Cambridge university press.

Jones, A., Thomson, D., Hort, M., and Devenish, B. (2007). The uk met office's next-generation atmospheric dispersion model, name iii. Air Pollution Modeling and its Application XVII, pages 580-589.

Keats, A., Yee, E., and Lien, F.-S. (2007). Bayesian inference for source determination with applications to a complex urban environment. Atmospheric environment, 41(3):465-479.

Kowadlo, G. and Russell, R. A. (2008). Robot odor localization: a taxonomy and survey. The International Journal of Robotics Research, 27(8):869-894.

Ladosz, P., Coombes, M., Smith, J., and Hutchinson, M. (2019). A generic ros based system for rapid development and testing of algorithms for autonomous ground and aerial vehicles. In Robot Operating System (ROS), pages 113-153. Springer.

Li, J.-G., Meng, Q.-H., Wang, Y., and Zeng, M. (2011). Odor source localization using a mobile robot in outdoor airflow environments with a particle filter algorithm. Autonomous Robots, 30(3):281-292.

Lilienthal, A. J., Reggente, M., Trincavelli, M., Blanco, J. L., and Gonzalez, J. (2009). A statistical approach to gas distribution modelling with mobile robots-the kernel $\mathrm{dm}+\mathrm{v}$ algorithm. In Intelligent Robots and Systems, 2009. IROS 2009. IEEE/RSJ International Conference on, pages 570-576. IEEE.

Luo, B., Meng, Q.-H., Wang, J.-Y., and Zeng, M. (2018). A flying odor compass to autonomously locate the gas source. IEEE Transactions on Instrumentation and Measurement, 67(1):137-149.

Marques, L. (2011). Good experimental methodologies for mobile robot olfaction. In Proceedings of the Robotics: Science and Systems Conference (RSS2009), Workshop on Good Experimental Methodology in Robotics, Seattle, WA, USA, volume 28, pages 291-294.

Masson, J.-B. (2013). Olfactory searches with limited space perception. Proceedings of the National Academy of Sciences, 110(28):11261-11266. 
Mastin, L., Guffanti, M., Servranckx, R., Webley, P., Barsotti, S., Dean, K., Durant, A., Ewert, J., Neri, A., Rose, W. I., et al. (2009). A multidisciplinary effort to assign realistic source parameters to models of volcanic ash-cloud transport and dispersion during eruptions. Journal of Volcanology and Geothermal Research, 186(1):10-21.

Moraud, E. M. and Martinez, D. (2010). Effectiveness and robustness of robot infotaxis for searching in dilute conditions. Frontiers in neurorobotics, 4.

Neumann, P. P. and Bartholmai, M. (2015). Real-time wind estimation on a micro unmanned aerial vehicle using its inertial measurement unit. Sensors and Actuators A: Physical, 235:300-310.

Neumann, P. P., Hernandez Bennetts, V., Lilienthal, A. J., Bartholmai, M., and Schiller, J. H. (2013). Gas source localization with a micro-drone using bio-inspired and particle filter-based algorithms. Advanced Robotics, 27(9):725-738.

Neumann, P. P., Kohlhoff, H., Hüllmann, D., Lilienthal, A. J., and Kluge, M. (2017). Bringing mobile robot olfaction to the next dimensionuav-based remote sensing of gas clouds and source localization. In Robotics and Automation (ICRA), 2017 IEEE International Conference on, pages 3910-3916. IEEE.

Okumura, T., Suzuki, K., Fukuda, A., Kohama, A., Takasu, N., Ishimatsu, S., and Hinohara, S. (1998). The tokyo subway sarin attack: disaster management, part 1: Community emergency response. Academic Emergency Medicine, 5(6):613-617.

Pasquill, F. (1961). The estimation of the dispersion of windborne material. Met. Mag., 90:33.

Rao, K. S. (2005). Uncertainty analysis in atmospheric dispersion modeling. Pure and applied geophysics, 162(10):1893-1917.

Rao, K. S. (2007). Source estimation methods for atmospheric dispersion. Atmospheric Environment, 41(33):6964-6973.

Redwood, M. (2011). Source term estimation and event reconstruction: a survey. Contract Report for $A D M L C, 51790$.

Ristic, B., Arulampalam, S., and Gordon, N. J. (2004). Beyond the Kalman filter: Particle filters for tracking applications. Artech house.

Ristic, B., Morelande, M., and Gunatilaka, A. (2010). Information driven search for point sources of gamma radiation. Signal Processing, 90(4):1225-1239. 
Ristic, B., Skvortsov, A., and Gunatilaka, A. (2016). A study of cognitive strategies for an autonomous search. Information Fusion, 28:1-9.

Roldán, J. J., Joossen, G., Sanz, D., del Cerro, J., and Barrientos, A. (2015). Mini-uav based sensory system for measuring environmental variables in greenhouses. Sensors, 15(2):3334-3350.

Senocak, I., Hengartner, N. W., Short, M. B., and Daniel, W. B. (2008). Stochastic event reconstruction of atmospheric contaminant dispersion using bayesian inference. Atmospheric Environment, 42(33):77187727.

Shraiman, B. I. and Siggia, E. D. (2000). Scalar turbulence. Nature, 405(6787):639-646.

Singh, S. K., Sharan, M., and Issartel, J.-P. (2015). Inverse modelling methods for identifying unknown releases in emergency scenarios: an overview. International Journal of Environment and Pollution, $57(1-2): 68-91$.

Šmídl, V. and Hofman, R. (2013). Tracking of atmospheric release of pollution using unmanned aerial vehicles. Atmospheric Environment, 67:425-436.

Smith, B., John, G., Stark, B., Christensen, L. E., and Chen, Y. (2016). Applicability of unmanned aerial systems for leak detection. In Unmanned Aircraft Systems (ICUAS), 2016 International Conference on, pages 1220-1227. IEEE.

Smith, B. J., John, G., Christensen, L. E., and Chen, Y. (2017). Fugitive methane leak detection using suas and miniature laser spectrometer payload: System, application and groundtruthing tests. In Unmanned Aircraft Systems (ICUAS), 2017 International Conference on, pages 369-374. IEEE.

Thanabalasingham, T., Beckett, M., and Murray, V. (1991). Hospital response to a chemical incident: report on casualties of an ethyldichlorosilane spill. BMJ: British Medical Journal, 302(6768):101.

Vergassola, M., Villermaux, E., and Shraiman, B. I. (2007). infotaxis as a strategy for searching without gradients. Nature, 445(7126):406-409.

Villa, T. F., Salimi, F., Morton, K., Morawska, L., and Gonzalez, F. (2016). Development and validation of a uav based system for air pollution measurements. Sensors, 16(12):2202.

Voges, N., Chaffiol, A., Lucas, P., and Martinez, D. (2014). Reactive searching and infotaxis in odor source localization. PLoS computational biology, 10(10):e1003861. 
Wang, Y., Huang, H., Huang, L., and Ristic, B. (2017). Evaluation of bayesian source estimation methods with prairie grass observations and gaussian plume model: A comparison of likelihood functions and distance measures. Atmospheric environment, 152:519-530.

Wiedemann, T., Manss, C., Shutin, D., Lilienthal, A. J., Karolj, V., and Viseras, A. (2017). Probabilistic modeling of gas diffusion with partial differential equations for multi-robot exploration and gas source localization. In Mobile Robots (ECMR), 2017 European Conference on, pages 1-7. IEEE.

Winiarek, V., Bocquet, M., Duhanyan, N., Roustan, Y., Saunier, O., and Mathieu, A. (2014). Estimation of the caesium-137 source term from the fukushima daiichi nuclear power plant using a consistent joint assimilation of air concentration and deposition observations. Atmospheric environment, 82:268-279.

Yee, E. (2017). Automated computational inference engine for bayesian source reconstruction: Application to some detections/non-detections made in the ctbt international monitoring system. Applied Mathematical Sciences, 11(32):1581-1618. 